\title{
Risk measures based on benchmark loss distributions
}

\author{
Valeria Bignozzi ${ }^{1}$, Matteo Burzoni ${ }^{2}$, Cosimo Munari ${ }^{3}$
}

November 19, 2018

\begin{abstract}
We introduce a class of quantile-based risk measures that generalize Value at Risk (VaR) and, likewise Expected Shortfall (ES), take into account both the frequency and the severity of losses. Under VaR a single confidence level is assigned regardless of the size of potential losses. We allow for a range of confidence levels that depend on the loss magnitude. The key ingredient is a benchmark loss distribution (BLD), i.e. a function that associates to each potential loss a maximal acceptable probability of occurrence. The corresponding risk measure, called Loss VaR (LVaR), determines the minimal capital injection that is required to align the loss distribution of a risky position to the target BLD. By design, one has full flexibility in the choice of the BLD profile and, therefore, in the range of relevant quantiles. Special attention is given to piecewise constant functions and to tail distributions of benchmark random losses, in which case the acceptability condition imposed by the BLD boils down to first-order stochastic dominance. We provide a comprehensive study of the main finance theoretical and statistical properties of LVaR with a focus on their comparison with VaR and ES. Merits and drawbacks are discussed and applications to capital adequacy, portfolio risk management and catastrophic risk are presented.
\end{abstract}

Keywords: risk measures, loss distributions, tail risk, capital adequacy, portfolio management, catastrophic risk, robustness, backtestability.

JEL classification: D81, G32.

Acknowledgements: This research started while the second and third author were visiting the Department of Mathematics of the University of Milan and the Department of Statistics and Quantitative Methods of the University of Milano-Bicocca supported by the ACRI (Associazione Casse Risparmio Italiane) Research Prize 2017. The second author acknowledges financial support from the ETH Foundation. We would like to thank Pablo Koch-Medina, Andreas Tsanakas, and Ruodu Wang for useful discussions and suggestions on an earlier version of this paper. The paper is Swiss Finance Institute Research Paper No. 18-48 and received a Best Paper Award at the 10th Conference in Actuarial Science \& Finance on Samos held in May-June 2018.

\section{Introduction}

Much of the debate about capital adequacy and solvency regulation over the last years has been dominated by Value at Risk (VaR) and Expected Shortfall (ES). The discussion is, nowadays, more heated than ever since VaR and ES are currently adopted in different regulatory regimes and their relative merits and drawbacks, which have been mainly investigated from a theoretical perspective, can be eventually evaluated against their concrete implementation.

\footnotetext{
${ }^{1}$ Department of Statistics and Quantitative Methods, University of Milano-Bicocca, valeria.bignozzi@unimib.it

${ }^{2}$ Department of Mathematics, ETH Zurich, matteo.burzoni@math.ethz.ch

${ }^{3}$ Center for Finance and Insurance and Swiss Finance Institute, University of Zurich, cosimo.munari@bf .uzh.ch
} 


\section{Value at Risk}

As is well-known, VaR was introduced as part of the market risk management toolbox developed by JP Morgan and released under the name RiskMetrics in 1994. A personal retrospective on the birth of VaR is provided in Guldimann (2000). The new risk measure soon became the industry standard and was eventually recognized at a regulatory level when the Basel Committee on Banking Supervision (BCBS) adopted it as one of the key ingredients of the second Basel Accord in 2004, usually known as Basel 2. The centrality of VaR was confirmed by Basel 3 in 2010-2011. The transition to a risk-sensitive solvency framework in the insurance industry, which culminated in the enforcement of Solvency 2 within the European Union in 2009, has assigned to VaR a central role also in the insurance regulatory world.

To recall the definition of $\mathrm{VaR}$, fix a probability space $(\Omega, \mathcal{F}, \mathbb{P})$ and let $X$ be a random variable representing a financial loss at a given future point in time (positive values of $X$ are understood as losses and negative values as profits). The $\operatorname{VaR}$ of $X$ at level $p \in(0,1]$ is defined by

$$
\operatorname{VaR}_{p}(X):=\inf \{m \in \mathbb{R} ; \mathbb{P}(X \leq m) \geq p\} .
$$

Being equal to the (left) $p$-quantile of $X$, the quantity $\operatorname{VaR}_{p}(X)$ can be naturally interpreted as the best outcome of $X$ out of the worst $100(1-p) \%$ of outcomes. Equivalently, $\operatorname{VaR}_{p}(X)$ can be interpreted as the minimal amount of capital that has to be raised and held in cash in order to absorb future losses in at least $100 p \%$ of cases. In this sense, one often says that $\operatorname{VaR}_{p}(X)$ quantifies the risk of the position $X$ as the cost of making the risk profile of $X$ "acceptable" in the following sense:

$$
X \text { is acceptable } \Longleftrightarrow \operatorname{VaR}_{p}(X) \leq 0 \Longleftrightarrow \mathbb{P}(X>0) \leq 1-p .
$$

In spite of being the most widely used risk measure in practice, VaR has been strongly criticized in the literature for two main reasons. On the one hand, VaR depends only on the frequency of losses and not on their severity and, hence, may fail to capture tail risk in an appropriate way. On the other hand, VaR is neither convex nor subadditive and thus fails to be a coherent risk measure in the sense of Artzner et al. (1999). In particular, this implies that VaR may penalize diversification and risk decentralization by not allowing to control the risk of an aggregate position in terms of the risk of its individual components.

\section{Expected Shortfall}

Among the coherent risk measures that have been proposed as alternatives to VaR, the most successful one is certainly ES as defined and first comprehensively studied by Acerbi and Tasche (2002). After some initial skepticism, ES has eventually encountered the favor of many practitioners and obtained the seal of approval also by regulators. In the insurance world, ES has been adopted since 2011 as the reference risk metric of the Swiss Solvency Test, the regulatory framework for insurance companies in Switzerland. In the banking world, the BCBS announced a shift from VaR to ES in the assessment of market risk to be implemented in the forthcoming Basel 4. Recall that the ES of a position $X$ at level $p \in(0,1)$ is given by

$$
\operatorname{ES}_{p}(X):=\frac{1}{1-p} \int_{p}^{1} \operatorname{VaR}_{q}(X) \mathrm{d} q
$$

It is well-known that, up to a correction term that accounts for a discontinuity in the distribution of $X$, the quantity $\operatorname{ES}_{p}(X)$ can be expressed as the conditional expectation of $X$ beyond the (left) $p$-quantile. Since we can always write

$$
\operatorname{ES}_{p}(X)=\inf \left\{m \in \mathbb{R} ; \operatorname{ES}_{p}(X-m) \leq 0\right\},
$$


the quantity $\mathrm{ES}_{p}(X)$ can also be interpreted as the minimal amount of capital that has to be raised and held in cash in order to ensure that, on average, $X$ does not incur a loss in the worst $100(1-p) \%$ of outcomes. As a result, $\mathrm{ES}_{p}(X)$ quantifies the risk of the position $X$ as the cost of making the risk profile of $X$ "acceptable" in the following sense $\left(F_{X}\right.$ is the distribution function of $X)$ :

$$
X \text { is acceptable } \Longleftrightarrow \operatorname{ES}_{p}(X) \leq 0 \stackrel{F_{X}}{\Longleftrightarrow \text { continuous }} \mathbb{E}\left[X \mid X \geq \operatorname{VaR}_{p}(X)\right] \leq 0 .
$$

In words, a position is acceptable under ES if, on average, it does not incur a loss in the tail beyond a certain pre-specified quantile.

\section{Tail risk under VaR and ES}

As said above, tail risk is captured by VaR by imposing an upper bound on the loss probability. Since, by definition, VaR depends only on the frequency of losses but not on their severity, one could in principle accumulate arbitrary loss peaks beyond the chosen quantile without being detected by VaR. This "blindness" of VaR to the tail of the loss distribution arguably constitutes the most fundamental deficiency of VaR and its undesirable financial implications have been analyzed by a vast literature, see e.g. Artzner et al. (1999), Daníelsson et al. (2001), Acerbi and Tasche (2002), Albanese and Lawi (2004), Galichon (2010), Jarrow (2013), Embrechts et al. (2018), Wang (2016), Weber (2018).

Differently from VaR, ES depends both on the frequency and the severity of losses and captures tail risk by controlling the average loss in the tail of the distribution beyond a pre-specified quantile threshold. As illustrated by the following quote from the Consultative Document issued in May 2012 (see p. 20 in BCBS, 2012), tail risk was the key concern that ultimately led the BCBS to move away from VaR and adopt ES:

The current framework's reliance on VaR as a quantitative risk metric stems largely from historical precedent and common industry practice. This has been reinforced over time by the requirement to use VaR for regulatory capital purposes. However, a number of weaknesses have been identified with VaR, including its inability to capture "tail risk". The Committee therefore believes it is necessary to consider alternative risk metrics that may overcome these weaknesses. Expected shortfall [...] is an example of a risk metric that considers a broader range of potential outcomes than VaR. Unlike VaR, ES measures the riskiness of an instrument by considering both the size and likelihood of losses above a certain threshold [...]. In this way, ES accounts for tail risk in a more comprehensive manner.

We also refer to the Executive Summary of the Standards on Capital Requirements issued in January 2016 (see p. 1 in BCBS, 2016). A similar concern has been raised by the International Association of Insurance Supervisors (IAIS). In the Public Consultation Document on RiskBased Global Insurance Capital Standard issued in December 2014 (see p. 41 in IAIS, 2014) the IAIS provides a detailed comparison between VaR and ES and states that (here Tail-VaR stands for $\mathrm{ES})$ :

For many business lines, IAIGs [Internationally Active Insurance Groups] may be subject to infrequent, high-impact losses. Under VaR, insured losses with probability beyond the confidence threshold receive no risk charge, but do in the Tail-VaR. Thus Tail-VaR provides an incentive for IAIGs to mitigate even the more extreme losses, and for supervisors to see that they do so. 


\section{Loss VaR: A new perspective on tail risk}

The goal of this paper is to contribute to the ongoing research on quantitative risk management by introducing a class of risk measures that capture tail risk in a different way as compared to VaR and ES. The focus is on risk measures that allow to control, for every level $u \geq 0$, the probability of incurring losses higher than $u$ in line with the principle that higher losses should be tolerated with lower probability.

The ability to control loss peaks is one of the crucial aspects of external as well as internal risk management. On the one side, a regulator is not only concerned with monitoring the default probability of a financial institution but also with exerting control on the likelihood of certain recovery levels on the underlying liabilities. On the other side, a portfolio risk manager is often concerned with the risk of exceeding some critical loss levels, which may correspond, for instance, to some pre-specified fractions of the invested capital. In both cases, it is reasonable that the degree of tolerance for loss exceedances decreases with the size of the potential losses. It is important to observe that neither VaR nor ES capture the above aspect of tail risk. This is clear for VaR because, being a single quantile, VaR is completely blind to the tail beyond the chosen quantile level. As demonstrated in Proposition 3.4, this is the case for ES as well. The intuition is that, by averaging across the tail, ES cannot provide a tight control on the shape of the tail distribution and, specifically, on the probability of exceeding a certain loss threshold (besides the zero threshold).

The key ingredient of our approach is a benchmark loss distribution, i.e. an increasing and right-continuous function

$$
\alpha:[0, \infty) \rightarrow(0,1]
$$

that specifies, for each loss level $u$, a maximal probability with which losses exceeding $u$ are tolerated. The interpretation is that a loss exceeding $u$ is tolerated in at most $100(1-\alpha(u)) \%$ of cases. As said above, the underlying principle, which was inspired by ideas put forward in Frittelli et al. (2014), is that higher losses should be accepted with lower probability. This is reflected by the monotonicity assumption on $\alpha$. To every benchmark loss distribution $\alpha$ we associate a risk measure defined by

$$
\operatorname{LVaR}_{\alpha}(X):=\inf \{m \in \mathbb{R} ; \mathbb{P}(X-m \leq u) \geq \alpha(u) \text { for every } u \geq 0\},
$$

which we call Loss Value at Risk with benchmark function $\alpha$. The risk measure LVaR admits a clear interpretation in terms of capital requirements: The quantity $\operatorname{LVaR}_{\alpha}(X)$ represents the minimal amount of capital that has to be raised and held in cash in order to align the distribution of $X$ to the benchmark loss profile prescribed by $\alpha$. The corresponding acceptability condition thus boils down to:

$X$ is acceptable $\Longleftrightarrow \operatorname{LVaR}_{\alpha}(X) \leq 0 \Longleftrightarrow \mathbb{P}(X>u) \leq 1-\alpha(u) \quad \forall u \geq 0$.

As is easily seen, the acceptability criterion induced by LVaR can be naturally viewed as a strengthening of the standard criterion induced by VaR: Instead of defining acceptability by imposing an upper bound on the loss probability $\mathbb{P}(X>0)$ alone, we consider the whole spectrum of probabilities $\mathbb{P}(X>u)$ for every loss level $u$. Thus, by construction, $\mathrm{LVaR}_{\alpha}$ is more conservative than $\mathrm{VaR}$ at the reference confidence level $\alpha(0)$, i.e. we always have

$$
\operatorname{LVaR}_{\alpha}(X) \geq \operatorname{VaR}_{\alpha(0)}(X) .
$$

We show in Proposition 3.7 that LVaR can be equivalently expressed as

$$
\operatorname{LVaR}_{\alpha}(X)=\sup _{u \geq 0}\left(\operatorname{VaR}_{\alpha(u)}(X)-u\right) .
$$


From the above formula it is clear that $\mathrm{LVaR}_{\alpha}$ depends upon the entire loss tail of $X$ between the $\alpha(0)$-quantile and the $\alpha(\infty)$-quantile, where $\alpha(\infty)=\lim _{u \rightarrow \infty} \alpha(u)$. The relevant portion of the loss distribution will be determined by the choice of the benchmark function. In particular, taking $\alpha(\infty)=1$ will make $\mathrm{LVaR}_{\alpha}$ depend on the entire loss tail beyond the $\alpha(0)$-quantile. In the extreme case of a constant benchmark function, $\mathrm{LVaR}_{\alpha}$ reduces to $\mathrm{VaR}_{\alpha(0)}$. In this case, we are indifferent with respect to the severity of losses and only focus on their frequency.

\section{Comparison with VaR and ES}

Every risk measure captures tail risk in a particular way and, by doing so, it fails to encompass some of its multiple facets. As argued above, LVaR captures tail risk in a more comprehensive way than VaR and in a different way as compared to ES. Hence, the choice of the risk measure will ultimately depend on the aspect(s) of tail risk that one deems to be more important. It is, however, clear that a thorough comparison between LVaR and VaR and especially between LVaR and ES should not take into account tail risk only. To this end, we provide a comprehensive study of the properties of LVaR with a view towards the most recent developments of risk measure theory in Sections 4 and 5. In particular, we show that, similarly to VaR, LVaR fails to be subadditive and convex and, therefore, it does not qualify as a coherent risk measure. Nevertheless, it is worth highlighting that the standard examples showing that VaR is not subadditive heavily exploit the tail blindness of VaR and may not work under LVaR (especially when the whole tail beyond the $\alpha(0)$-quantile is taken into account). On the other hand, LVaR satisfies two properties that are fulfilled by VaR but not by ES, namely surplus invariance and (the standard form of) statistical robustness. We also discuss some potential ways to backtest LVaR.

While $\mathrm{LVaR}_{\alpha}$ is always more conservative than $\operatorname{VaR}_{\alpha(0)}$, our examples illustrate that there is generally no relation between $\mathrm{LVaR}_{\alpha}$ and $\mathrm{ES}_{\alpha(0)}$ and, depending on the underlying distribution, $\mathrm{LVaR}_{\alpha}$ may be more or less conservative than $\mathrm{ES}_{\alpha(0)}$. This is true regardless of whether the entire loss tail is taken into account under $\mathrm{LVaR}_{\alpha}$ or not. In this sense, the differences

$$
\operatorname{LVaR}_{\alpha}(X)-\operatorname{VaR}_{\alpha(0)}(X) \quad \text { and } \operatorname{LVaR}_{\alpha}(X)-\mathrm{ES}_{\alpha(0)}(X)
$$

can be used as an indicator of how far VaR and ES are from controlling the probability of loss exceedances as specified by the benchmark loss distribution. Moreover, it is important to highlight that there are applications where using ES might be undesirable or potentially unfeasible due to the presence, e.g., of infinite-mean loss distributions. In this case, LVaR provides a concrete alternative to VaR which, likewise ES, takes into account both the frequency and the severity of losses. We illustrate this point in the context of Pareto-tailed risks in Section 6. Finally, we wish to emphasize that LVaR allows full flexibility in the choice of the benchmark loss distribution. This is important for two main reasons. First, depending on the area of application, the shape of a "benchmark distribution" may be radically different, ranging from close-to-normal distributions to very skewed and heavy-tailed distributions. Therefore, differently from VaR and ES, LVaR can be easily tailored to the "tail class" of the specific application. Second, even within the same field, two risk managers may impose the same (regulatory) bound on the loss probability $\mathbb{P}(X>0)$ and yet have different benchmark loss profiles. In this case, the choice of $\alpha$ allows to incorporate the risk appetite of the individual risk manager while, at the same time, respecting some externally imposed requirement. We refer to Section 6 for a discussion on concrete choices of $\alpha$. 


\section{Structure of the paper}

The paper is structured as follows. After recalling some background notions in Section 2, we introduce acceptance sets and risk measures based on benchmark loss distributions in Section 3 . We provide a detailed study of their finance theoretical properties in Section 4 and of statistical properties in Section 5. We illustrate applicability in Section 6 by focusing on a variety of familiar distributions and comparing the outcomes of LVaR with those of VaR and ES. Section 7 concludes the paper.

\section{Notation and terminology}

Throughout the paper we fix a nonatomic probability space $(\Omega, \mathcal{F}, \mathbb{P})$ and denote by $\mathcal{X}$ the vector space of all random variables over $(\Omega, \mathcal{F}, \mathbb{P})$. As usual, we do not distinguish between two random variables that coincide $\mathbb{P}$-almost surely. In this paper we follow the convention to represent losses by positive amounts and profits by negative amounts. In particular, for any random variable $X$ the positive and negative part, $X^{+}:=\max (X, 0)$ and $X^{-}:=\max (-X, 0)$, correspond to the loss and profit profile of $X$, respectively. We say that $X$ is bounded whenever $\mathbb{P}(|X| \leq c)=1$ for some $c \geq 0$. Moreover, $X$ and $Y$ are said to be comonotone whenever there exist a random variable $Z$ and increasing functions $f, g: \mathbb{R} \rightarrow \mathbb{R}$ such that $X=f(Z)$ and $Y=g(Z)$.

The distribution function of $X$ is the increasing and right-continuous map $F_{X}: \mathbb{R} \rightarrow[0,1]$ given by $F_{X}(x):=\mathbb{P}(X \leq x)$, for all $x \in \mathbb{R}$. Recall that any increasing and right-continuous function $F: \mathbb{R} \rightarrow[0,1]$ with infimum equal to 0 and supremum equal to 1 is, by nonatomicity, the distribution function of a suitable random variable. We write $X \sim Y$ whenever $F_{X}(x)=F_{Y}(x)$ for all $x \in \mathbb{R}$. In addition, we consider the standard relations on $\mathcal{X}$ defined by

$$
X \leq Y \Longleftrightarrow \mathbb{P}(X \leq Y)=1 \quad \text { and } \quad X \leq_{s t} Y \Longleftrightarrow F_{X}(x) \geq F_{Y}(x), \forall x \in \mathbb{R} .
$$

In this paper we consider risk measures in the spirit of Artzner et al. (1999). After specifying an acceptance set $\mathcal{A} \subset \mathcal{X}$, which represents the set of positions that are deemed acceptable from an internal or external regulatory perspective, we define the risk measure associated to $\mathcal{A}$ as the $\operatorname{map} \rho_{\mathcal{A}}: \mathcal{X} \rightarrow[-\infty,+\infty]$ given by

$$
\rho_{\mathcal{A}}(X):=\inf \{m \in \mathbb{R} ; X-m \in \mathcal{A}\} .
$$

For every position $X$ the quantity $\rho_{\mathcal{A}}(X)$ represents, when finite, the minimal amount of capital that has to be raised and held in cash in order to align $X$ with the acceptability constraint imposed by $\mathcal{A}$. In this sense, $\rho_{\mathcal{A}}(X)$ can be naturally interpreted as a capital requirement. Of course, if $X$ is already acceptable in the first place, then $\rho_{\mathcal{A}}(X)$ is negative and can be interpreted as a capital amount that can be obtained by extracting cash from $X$ without losing acceptability. Due to their clear operational interpretation, the above risk measures are particularly suited to be employed in a capital adequacy framework. Note that, by definition, $\rho_{\mathcal{A}}$ satisfies the property of cash-additivity, i.e. for all $X \in \mathcal{X}$ and $m \in \mathbb{R}$ we have

$$
\rho_{\mathcal{A}}(X+m)=\rho_{\mathcal{A}}(X)+m .
$$

It is easy to verify that VaR and ES are special cases of risk measures of the form $\rho_{\mathcal{A}}$. The acceptance set associated with $\operatorname{VaR}$ at level $p \in(0,1]$ is

$$
\mathcal{A}_{\mathrm{VaR}}(p):=\left\{X \in \mathcal{X} ; \operatorname{VaR}_{p}(X) \leq 0\right\}=\{X \in \mathcal{X} ; \mathbb{P}(X \leq 0) \geq p\} .
$$


ES can be seen as a particular case of another risk measure, the so-called Range Value at Risk (RVaR), that will constitute a natural term of comparison for our risk measures. The RVaR of a position $X$ between the levels $0<p<q \leq 1$ is defined by

$$
\operatorname{RVaR}_{p, q}(X):=\frac{1}{q-p} \int_{p}^{q} \operatorname{VaR}_{r}(X) \mathrm{d} r .
$$

One can verify that $\mathrm{RVaR}_{p, q}$ is cash-additive and the corresponding acceptance set is

$$
\mathcal{A}_{\mathrm{RVaR}}(p, q):=\left\{X \in \mathcal{X} ; \operatorname{RVaR}_{p, q}(X) \leq 0\right\} .
$$

Similarly to acceptability based on ES, a position is acceptable under RVaR provided there is no loss on average in the portion of the loss distribution between the left $p$-quantile and the left q-quantile. RVaR was introduced in Cont et al. (2010) as a "robust" alternative to ES; see Section 5 for more details.

Remark 2.1. The theory of cash-additive risk measures is thoroughly presented in Föllmer and Schied (2016). In addition, we refer to McNeil et al. (2015) for more information about VaR and ES and their practical relevance. Note that, instead of cash, one could generalize the risk measure in (1) by using any (riskless or risky) reference asset as the vehicle to reach acceptability. This possibility was already mentioned in the original paper by Artzner et al. (1999) and has been investigated in Artzner et al. (2009) and more recently in Farkas et al. (2014b).

\section{Risk measures based on benchmark loss distributions}

In this section we introduce a class of quantile-based risk measures that generalize VaR and, likewise ES, allow to take into account both the frequency and severity of potential losses. In line with the framework of Artzner et al. (1999), we start by specifying a notion of acceptability and then proceed to consider the associated risk measure.

The starting point is to fix some benchmark loss distribution (BLD), i.e. an increasing and right-continuous function

$$
\alpha:[0, \infty) \rightarrow(0,1]
$$

which assigns to each loss level $u \geq 0$ a certain probability weight $\alpha(u) \in(0,1]$. Note that, even though we use the term "distribution", the function $\alpha$ need not qualify as a distribution function in a technical sense (even if we set it equal to zero on the negative half-line) since we do not assume the supremum of $\alpha$ to be equal to 1 . For later use we define the functions $\alpha_{-}^{-1}, \alpha_{+}^{-1}:[0,1] \rightarrow[0, \infty]$ by

$$
\alpha_{-}^{-1}(p):=\inf \{u \geq 0 ; \alpha(u) \geq p\}, \quad \alpha_{+}^{-1}(p):=\inf \{u \geq 0 ; \alpha(u)>p\} .
$$

Moreover, we adopt the convenient notation

$$
\underline{\alpha}:=\inf _{u \geq 0} \alpha(u)=\alpha(0), \quad \bar{\alpha}:=\sup _{u \geq 0} \alpha(u)=\lim _{u \rightarrow \infty} \alpha(u) .
$$

To every benchmark loss distribution $\alpha$ we associate an acceptance set as follows.

Definition 3.1. The acceptance set based on a BLD $\alpha$ is defined by

$$
\mathcal{A}_{\alpha}:=\left\{X \in \mathcal{X} ; F_{X}(u) \geq \alpha(u), \forall u \geq 0\right\} .
$$


The financial interpretation of the acceptance set $\mathcal{A}_{\alpha}$ is similar to that of acceptability based on VaR: A position $X$ is acceptable whenever, for every loss level $u$, the probability of incurring a loss beyond level $u$ does not exceed the threshold $1-\alpha(u)$. As such, the above acceptability criterion can be viewed as a natural generalization of the VaR criterion, which corresponds to a constant benchmark loss distribution. The connection with $\mathrm{VaR}$ is clearly highlighted in the following proposition.

Proposition 3.2. For every $B L D \alpha$ the acceptance set $\mathcal{A}_{\alpha}$ admits the following representations:

(a) $\mathcal{A}_{\alpha}=\left\{X \in \mathcal{X} ; X-u \in \mathcal{A}_{\mathrm{VaR}}(\alpha(u)), \forall u \geq 0\right\}$.

(b) $\mathcal{A}_{\alpha}=\left\{X \in \mathcal{X} ; \operatorname{VaR}_{\alpha(u)}(X) \leq u, \forall u \geq 0\right\}$.

In particular, we have $\mathcal{A}_{\mathrm{VaR}}(\bar{\alpha}) \subseteq \mathcal{A}_{\alpha} \subseteq \mathcal{A}_{\mathrm{VaR}}(\underline{\alpha})$.

Proof. For every $u \geq 0$ we have $F_{X}(u) \geq \alpha(u)$ if, and only if, $F_{X-u}(0) \geq \alpha(u)$, which is equivalent to $X-u \in \mathcal{A}_{\mathrm{VaR}}(\alpha(u))$. This establishes the representation (a). The equivalence between $(a)$ and $(b)$ follows directly from the cash-additivity of VaR. To establish the left-hand side inclusion, take $X \in \mathcal{A}_{\mathrm{VaR}}(\bar{\alpha})$ and observe that

$$
F_{X}(u) \geq F_{X}(0) \geq \bar{\alpha} \geq \alpha(u)
$$

for all $u \geq 0$. This shows that $X \in \mathcal{A}_{\alpha}$ and proves the inclusion. The right-hand side inclusion follows immediately from (a) once we recall that $\underline{\alpha}=\alpha(0)$.

The acceptability criterion based on $\mathcal{A}_{\alpha}$ can be easily reformulated in the language of (first order) stochastic dominance whenever the benchmark loss distribution represents the right tail of a genuine distribution function.

Proposition 3.3. For every $B L D \alpha$ with $\bar{\alpha}=1$ the acceptance set $\mathcal{A}_{\alpha}$ admits the representation

$$
\mathcal{A}_{\alpha}=\left\{X \in \mathcal{X} ; X \leq_{s t} L\right\}
$$

where $L \in \mathcal{X}$ is any positive random variable satisfying $F_{L}(u)=\alpha(u)$ for $u \geq 0$.

Proof. Since $\alpha$ is increasing and right-continuous, the assumption $\bar{\alpha}=1$ implies, by nonatomicity, the existence of a random variable $L \in \mathcal{X}$ such that $F_{L}(u)=0$ for $u \in(-\infty, 0)$ and $F_{X}(u)=\alpha(u)$ for $u \in[0, \infty)$. The corresponding representation is immediate to verify.

By definition, the acceptability condition given by $\mathcal{A}_{\alpha}$ captures tail risk by imposing an upper bound on the probability of loss exceedances. This bound is tighter the higher the loss level. As mentioned in the introduction, neither VaR nor ES allow us to exercise the same control on tail risk. This is made clear in the following proposition, where we show that, for every loss level $u$, the loss probability $\mathbb{P}(X>u)$ can be bound from below uniformly on the positions $X$ that are acceptable under either VaR or ES at level $p$ only by the constant $1-p$.

Proposition 3.4. For every $p \in(0,1)$ and every $u \geq 0$ we have

$$
\sup _{X \in \mathcal{A}_{\mathrm{VaR}}(p)} \mathbb{P}(X>u)=\sup _{X \in \mathcal{A}_{\mathrm{ES}}(p)} \mathbb{P}(X>u)=1-p .
$$


Proof. Fix $u \geq 0$ and note that

$$
\begin{aligned}
1-p & \geq \sup \left\{\mathbb{P}(X>0) ; X \in \mathcal{A}_{\mathrm{VaR}}(p)\right\} \\
& \geq \sup \left\{\mathbb{P}(X>u) ; X \in \mathcal{A}_{\mathrm{VaR}}(p)\right\} \\
& \geq \sup \left\{\mathbb{P}(X>u) ; X \in \mathcal{A}_{\mathrm{ES}}(p)\right\}
\end{aligned}
$$

Now, for every $n \in \mathbb{N}$ consider a position $X_{n} \in \mathcal{X}$ such that

$$
\mathbb{P}\left(X_{n}=n\right)=\left(1-\frac{1}{n}\right)(1-p) \quad \text { and } \quad \mathbb{P}\left(X_{n}=n-n^{2}\right)=1-\left(1-\frac{1}{n}\right)(1-p) .
$$

Note that for every $n \in \mathbb{N}$

$$
\mathrm{ES}_{p}\left(X_{n}\right)=\frac{1}{1-p}\left(\left(\frac{1-p}{n}\right)\left(n-n^{2}\right)+\left(1-\frac{1}{n}\right)(1-p) n\right)=0 .
$$

Since $\mathbb{P}\left(X_{n}>u\right)=\mathbb{P}\left(X_{n}=n\right)$ for every $n>u$, we get

$$
\lim _{n \rightarrow \infty} \mathbb{P}\left(X_{n}>u\right)=\lim _{n \rightarrow \infty}\left(1-\frac{1}{n}\right)(1-p)=1-p .
$$

This proves the desired assertion.

The remainder of the paper will be devoted to the study of the risk measures associated to acceptance sets based on benchmark loss distributions.

Definition 3.5. The Loss VaR with $B L D \alpha$ is the risk measure $\operatorname{LVaR}_{\alpha}: \mathcal{X} \rightarrow[-\infty,+\infty]$ given by

$$
\operatorname{LVaR}_{\alpha}(X):=\rho_{\mathcal{A}_{\alpha}}(X)=\inf \left\{m \in \mathbb{R} ; X-m \in \mathcal{A}_{\alpha}\right\}
$$

In line with the financial interpretation of risk measures of the form $\rho_{\mathcal{A}}$, the quantity $\operatorname{LVaR}_{\alpha}(X)$ represents for every position $X$ the minimal amount of capital that has to be raised and held in cash to pass the acceptability test prescribed by $\mathcal{A}_{\alpha}$.

Remark 3.6. It is immediate to see that the acceptance set $\mathcal{A}_{\alpha}$ is radially closed along 1 in the sense that, for every $X \in \mathcal{X}$ and for every sequence $\left(m_{n}\right) \subset \mathbb{R}$ converging to $m \in \mathbb{R}$, we have $X-m \in \mathcal{A}_{\alpha}$ whenever $X-m_{n} \in \mathcal{A}_{\alpha}$ for every $n \in \mathbb{N}$. This clearly implies that

$$
\mathcal{A}_{\alpha}=\left\{X \in \mathcal{X} ; \operatorname{LVaR}_{\alpha}(X) \leq 0\right\}
$$

This fact will be repeatedly used without explicit reference in the sequel.

We provide a useful representation of $\mathrm{LVaR}_{\alpha}$ based on the representation of the acceptance set $\mathcal{A}_{\alpha}$ established in Proposition 3.2. This reformulation highlights, from a different angle, that $\mathrm{LVaR}_{\alpha}$ is more conservative than $\operatorname{VaR}_{\underline{\alpha}}$.

Proposition 3.7. For every $B L D \alpha$ the risk measure $\mathrm{LVaR}_{\alpha}$ admits the following representations:

$$
\operatorname{LVaR}_{\alpha}(X)=\sup _{u \geq 0}\left(\operatorname{VaR}_{\alpha(u)}(X)-u\right)=\sup _{p \in[\underline{\alpha}, \bar{\alpha}]}\left(\operatorname{VaR}_{p}(X)-\alpha_{-}^{-1}(p)\right) .
$$

In particular, we have

$$
\operatorname{VaR}_{\underline{\alpha}}(X) \leq \operatorname{LVaR}_{\alpha}(X) \leq \operatorname{VaR}_{\bar{\alpha}}(X) .
$$


Proof. Fix an arbitrary $X \in \mathcal{X}$. To show the first representation note that

$$
\begin{aligned}
\operatorname{LVaR}_{\alpha}(X) & =\inf \left\{m \in \mathbb{R} ; X-m \in \mathcal{A}_{\alpha}\right\} \\
& =\inf \left\{m \in \mathbb{R} ; \operatorname{VaR}_{\alpha(u)}(X-m) \leq u, \forall u \geq 0\right\} \\
& =\inf \left\{m \in \mathbb{R} ; \operatorname{VaR}_{\alpha(u)}(X)-u \leq m, \forall u \geq 0\right\} \\
& =\sup _{u \geq 0}\left(\operatorname{VaR}_{\alpha(u)}(X)-u\right),
\end{aligned}
$$

where we have used (b) in Proposition 3.2 in the second equality and the cash-additivity of VaR in the third one. To establish the second representation, take $u \geq 0$ and set $p=\alpha(u)$. Since $\alpha_{-}^{-1}(p) \leq u$, we infer that

$$
\operatorname{VaR}_{\alpha(u)}(X)-u \leq \operatorname{VaR}_{p}(X)-\alpha_{-}^{-1}(p) .
$$

This yields the inequality " $\leq$ " in the second representation in (2). Conversely, take $p \in[\underline{\alpha}, \bar{\alpha}]$ and assume that $\alpha_{-}^{-1}(p)<\infty$. Then, set $u=\alpha_{-}^{-1}(p)$ and note that $\alpha(u) \geq p$ by right-continuity of $\alpha$. As a result, we obtain

$$
\operatorname{VaR}_{\alpha(u)}(X)-u \geq \operatorname{VaR}_{p}(X)-\alpha_{-}^{-1}(p) .
$$

The preceding inequality trivially holds for every $u \geq 0$ if $\alpha_{-}^{-1}(p)=\infty$. This shows the inequality " $\geq$ " in the second representation in (2). The inequalities in (3) follow directly from the inclusions in Proposition 3.2.

In the case that $\alpha$ qualifies as the right tail of a genuine distribution function we obtain the following nice reformulation of $\mathrm{LVaR}_{\alpha}$.

Corollary 3.8. For every $B L D \alpha$ with $\bar{\alpha}=1$ the risk measure $\mathrm{LVaR}_{\alpha}$ admits the representation

$$
\operatorname{LVaR}_{\alpha}(X)=\sup _{p \in[\underline{\alpha}, 1]}\left(\operatorname{VaR}_{p}(X)-\operatorname{VaR}_{p}(L)\right)
$$

where $L \in \mathcal{X}$ is any positive random variable satisfying $F_{L}(u)=\alpha(u)$ for $u \geq 0$.

Remark 3.9. The above reformulations of LVaR are appealing from a computational point of view since they show that $\mathrm{LVaR}$ can be determined as the optimum value of a simple maximization problem. Note that, since the VaR component and the loss component in (2) display the opposite monotonicity as functions of the loss level $u$, the solution of the above maximization problem will critically depend on the random variable under consideration.

The following proposition provides a simple formula for LVaR when the benchmark loss distribution attains finitely many values. The result follows directly from the first representation of LVaR established in Proposition 3.7.

Proposition 3.10 (Piecewise constant BLD). Consider a BLD $\alpha$ and assume that

$$
\alpha(u)= \begin{cases}\alpha_{1} & \text { if } u_{1} \leq u<u_{2} \\ \alpha_{2} & \text { if } u_{2} \leq u<u_{3} \\ \vdots & \vdots \\ \alpha_{n} & \text { if } u \geq u_{n}\end{cases}
$$

for some $0<\alpha_{1}<\cdots<\alpha_{n} \leq 1$ and $0=u_{1}<\cdots<u_{n}$. Then, for every $X \in \mathcal{X}$ we have

$$
\operatorname{LVaR}_{\alpha}(X)=\max _{k=1, \ldots, n}\left(\operatorname{VaR}_{\alpha_{k}}(X)-u_{k}\right)
$$


In practice one often works with discrete random variables obtained through empirical distributions. The next result shows how to compute the risk measure LVaR in this case.

Proposition 3.11 (Discrete random variables). Assume $X \in \mathcal{X}$ attains the values $x_{1}<\cdots<$ $x_{n}$. Moreover, set $p_{0}=0$ and $p_{k}=F_{X}\left(x_{k}\right)$ for all $k=1, \ldots, n$. Then, for every $B L D \alpha$ we have

$$
\operatorname{LVaR}_{\alpha}(X)=\max _{k=1, \ldots, n}\left(x_{k}-\alpha_{+}^{-1}\left(p_{k-1}\right)\right) .
$$

Proof. Take first any $k \in\{1, \ldots, n\}$ and note that $\operatorname{VaR}_{p}(X)=x_{k}$ for all $p \in\left(p_{k-1}, p_{k}\right]$. Hence, it follows from Proposition 3.7 that

$$
\begin{aligned}
\operatorname{LVaR}_{\alpha}(X) & =\max _{k=1, \ldots, n} \sup _{p_{k-1}<p \leq p_{k}}\left(\operatorname{VaR}_{p}(X)-\alpha_{-}^{-1}(p)\right) \\
& =\max _{k=1, \ldots, n} \sup _{p_{k-1}<p \leq p_{k}}\left(x_{k}-\alpha_{-}^{-1}(p)\right) \\
& =\max _{k=1, \ldots, n}\left(x_{k}-\inf _{p_{k-1}<p \leq p_{k}} \alpha_{-}^{-1}(p)\right) \\
& =\max _{k=1, \ldots, n}\left(x_{k}-\alpha_{+}^{-1}\left(p_{k-1}\right)\right) .
\end{aligned}
$$

This establishes the desired formula.

By definition the quantity $\operatorname{LVaR}_{\alpha}(X)$ need not be finite for an arbitrary position $X$ and, hence, it is natural to ask under which conditions $\mathrm{LVaR}_{\alpha}$ is finitely valued. Note that, from a financial perspective, $\operatorname{LVaR}_{\alpha}(X)=\infty$ means that it is not possible to make the position $X$ acceptable at any finite cost. On the other side, $\operatorname{LVaR}_{\alpha}(X)=-\infty$ means that $X$ is acceptable and one can extract an arbitrary amount of cash from $X$ without losing acceptability.

Proposition 3.12. For every $B L D \alpha$ and every $X \in \mathcal{X}$ we have $\operatorname{LVaR}_{\alpha}(X)>-\infty$. Moreover, consider the following:

(a) The function $\alpha$ satisfies $\bar{\alpha}<1$.

(b) There exists $u_{0}>0$ such that $F_{X}(u) \geq \alpha(u)$ for all $u \geq u_{0}$.

(c) The distribution function $F_{X}$ satisfies

$$
\sup _{\substack{u \geq 0 \\ F_{X}(u)<\alpha(u)}} \inf _{\substack{z>u \\ F_{X}(z) \geq \alpha(z)}}(z-u)<\infty .
$$

(d) There exists $m>0$ such that $F_{X}(u+m) \geq \alpha(u)$ for all $u \geq 0$.

(e) $\operatorname{LVaR}_{\alpha}(X)<\infty$.

Then, $(a) \Longrightarrow(b) \Longrightarrow(c) \Longrightarrow(d) \Longleftrightarrow(e)$.

Proof. Fix an arbitrary $X \in \mathcal{X}$. To show the first assertion, recall that $\underline{\alpha}>0$ and note that

$$
\operatorname{LVaR}_{\alpha}(X) \geq \operatorname{VaR}_{\underline{\alpha}}(X)>-\infty
$$

by Proposition 3.7. Now, assume that (a) holds. In this case, we find $u_{0}>0$ large enough to have $F_{X}\left(u_{0}\right)>\bar{\alpha}$. Then, for every $u \geq u_{0}$ we easily obtain

$$
F_{X}(u) \geq F_{X}\left(u_{0}\right)>\bar{\alpha} \geq \alpha(u),
$$


showing that (b) holds. If (b) holds for some $u_{0}>0$, then $F_{X}\left(u_{0}\right) \geq \alpha\left(u_{0}\right)$ and every $u \geq 0$ with $F_{X}(u)<\alpha(u)$ must satisfy $u<u_{0}$. This yields

$$
\sup _{\substack{u \geq 0 \\ F_{X}(u)<\alpha(u)}} \inf _{\substack{z>u \\ F_{X}(z) \geq \alpha(z)}}(z-u) \leq u_{0}
$$

which implies (c). Next, assume that (c) holds and take $m>0$ larger than the supremum in (c). For every $u \geq 0$ we are in one of the following cases. If $F_{X}(u) \geq \alpha(u)$, then

$$
F_{X}(u+m) \geq F_{X}(u) \geq \alpha(u) .
$$

If otherwise $F_{X}(u)<\alpha(u)$, then we must find $z>u$ with $F_{X}(z) \geq \alpha(z)$ satisfying $z-u<m$, which implies

$$
F_{X}(u+m) \geq F_{X}(z) \geq \alpha(z) \geq \alpha(u) .
$$

Hence, we infer that $(d)$ holds. To establish the equivalence between $(d)$ and $(e)$, it suffices to observe that $\operatorname{LVaR}_{\alpha}(X)<\infty$ holds if, and only if, we find $m>0$ such that

$$
F_{X}(u+m)=F_{X-m}(u) \geq \alpha(u)
$$

for all $u \geq 0$. This concludes the proof of the proposition.

Remark 3.13. The implication $(c) \Longrightarrow(e)$ shows that we always have finiteness whenever the distance between any loss level where the distribution function $F_{X}$ is strictly below the benchmark loss distribution $\alpha$ and the first loss level where $F_{X}$ crosses $\alpha$ is uniformly bounded.

The following corollary is a direct consequence of Proposition 3.12 and is in line with the general results on finiteness from Farkas et al. (2014a).

Corollary 3.14. For every $B L D \alpha$ and every bounded $X \in \mathcal{X}$ we have $\operatorname{LVaR}_{\alpha}(X)<\infty$.

We conclude this section by highlighting the relation between LVaR and the risk measure $\Lambda$ VaR, which was introduced in Frittelli et al. (2014) and further studied in Burzoni et al. (2017). For an increasing and right-continuous function $\Lambda: \mathbb{R} \rightarrow[0,1]$ with $\Lambda(0) \geq 0$, called probability/loss function, we define the Lambda $V a R$ of a position $X$ by

$$
\Lambda \operatorname{VaR}(X):=\inf \left\{m \in \mathbb{R} ; F_{X}(u) \geq \Lambda(u), \forall u \geq m\right\} .
$$

Here, we have adapted the original definition to the sign convention of our paper. The aim of Frittelli et al. (2014) was to introduce a continuum of "degrees of acceptability" based on $\Lambda$ following the rationale that higher losses should be accepted with lower probabilities. In this sense, there is affinity between the benchmark loss distribution $\alpha$ and the probability/loss function $\Lambda$. However, in spite of their formal resemblance, there exists a critical financial difference between LVaR and $\Lambda$ VaR. While the risk coefficient $\operatorname{LVaR}_{\alpha}(X)$ is determined, in the spirit of Artzner et al. (1999), by translating the distribution of $X$ until we reach an acceptable configuration, the risk coefficient $\Lambda \operatorname{VaR}(X)$ is computed in a "static" way by looking at the maximal interval $[m, \infty)$ where the loss distribution of $X$ is above the probability/loss function $\Lambda$. In particular, contrary to LVaR, the "static" formulation of $\Lambda \mathrm{VaR}$ does not carry a clear operational interpretation from a capital adequacy perspective. In this sense, LVaR seems to constitute a valid alternative to $\Lambda \mathrm{VaR}$ in the framework of capital adequacy.

The next result highlights the relation between $\Lambda \mathrm{VaR}$ and LVaR when the benchmark function $\alpha$ is given in terms of the probability/loss function $\Lambda$. 
Proposition 3.15. Let $\Lambda: \mathbb{R} \rightarrow[0,1]$ be an increasing and right-continuous function with $\Lambda(0)>0$ and set $\alpha(u)=\Lambda(u)$ for all $u \geq 0$. Then, $\alpha$ is a BLD and for every $X \in \mathcal{X}$ the following hold:

(a) $\operatorname{LVaR}_{\alpha}(X) \leq 0 \Longleftrightarrow \Lambda \operatorname{VaR}(X) \leq 0$.

(b) $\left|\operatorname{LVaR}_{\alpha}(X)\right| \leq|\Lambda \operatorname{VaR}(X)|$.

Proof. (a) Assume first that $\Lambda \operatorname{VaR}(X) \leq 0$ and note that $F_{X}(u) \geq \alpha(u)$ for all $u \geq 0$ by right-continuity. This yields $\operatorname{LVaR}_{\alpha}(X) \leq 0$. Conversely, assume that $\Lambda \operatorname{VaR}(X)>0$. In this case, by definition, there exists $u \geq 0$ such that $F_{X}(u)<\alpha(u)$. This implies $X \notin \mathcal{A}_{\alpha}$ and thus $\operatorname{LVaR}_{\alpha}(X)>0$.

(b) Assume first that $\Lambda \operatorname{VaR}(X) \leq 0$. In this case, we have $\operatorname{LVaR}_{\alpha}(X) \leq 0$ and thus $\operatorname{LVaR}_{\alpha}(X) \in$ $\mathbb{R}$ by Proposition 3.12. In particular, it follows that $X-\operatorname{LVaR}_{\alpha}(X) \in \mathcal{A}_{\alpha}$ so that $F_{X}(u+$ $\left.\operatorname{LVaR}_{\alpha}(X)\right)=F_{X-\operatorname{LVaR}_{\alpha}(X)}(u) \geq \alpha(u)$ for all $u \geq 0$. As a result, we have $F_{X}(z) \geq \alpha(z-$ $\left.\operatorname{LVaR}_{\alpha}(X)\right) \geq \alpha(z)$ for all $z \geq \operatorname{LVaR}_{\alpha}(X)$, which yields $\Lambda \operatorname{VaR}(X) \leq \operatorname{LVaR}_{\alpha}(X)$. Next, assume that $0<\Lambda \operatorname{VaR}(X)<\infty$ and recall that $\operatorname{LVaR}_{\alpha}(X)>0$ in this case. Then, take an arbitrary $\varepsilon>0$ and observe that

$$
F_{X-\Lambda \operatorname{VaR}(X)-\varepsilon}(u)=F_{X}(\Lambda \operatorname{VaR}(X)+\varepsilon+u) \geq \Lambda(\Lambda \operatorname{VaR}(X)+\varepsilon+u) \geq \alpha(u)
$$

for every $u \geq 0$, showing that $\operatorname{LVaR}_{\alpha}(X) \leq \Lambda \operatorname{VaR}(X)+\varepsilon$. Since $\varepsilon$ was arbitrary, we conclude that $\operatorname{LVaR}_{\alpha}(X) \leq \Lambda \operatorname{VaR}(X)$.

Remark 3.16. Note that the inequality in (b) can be strict. To see this, take $X \in \mathcal{X}$ with strictly increasing distribution $F_{X}$ and, for a given $m>0$, define $\alpha(u)=\Lambda(u)=F_{X}(u+m)$ for $u \geq 0$. It is easy to see that $\operatorname{LVaR}_{\alpha}(X)=m$ but $\Lambda \operatorname{VaR}(X)=\infty$. Incidentally, this example also shows that the implication $(e) \Longrightarrow(c)$ in Proposition 3.12 does not hold in general.

\section{Finance theoretical properties}

In this section we consider a variety of standard properties of risk measures and discuss whether risk measures based on benchmark loss distributions satisfy them or not. The focus is on finance theoretical properties. The next section will be devoted to investigating statistical properties.

For a function $\rho: \mathcal{X} \rightarrow[-\infty,+\infty]$ we consider the following properties (we use the convention that adding $\infty$ to $-\infty$ yields $\infty)$ :

(i) monotonicity: $\rho(X) \leq \rho(Y)$ for all $X, Y \in \mathcal{X}$ such that $X \leq Y$.

(ii) stochastic monotonicity: $\rho(X) \leq \rho(Y)$ for all $X, Y \in \mathcal{X}$ such that $X \leq_{s t} Y$.

(iii) convexity: $\rho(\lambda X+(1-\lambda) Y) \leq \lambda \rho(X)+(1-\lambda) \rho(Y)$ for all $X, Y \in \mathcal{X}$ and $\lambda \in[0,1]$.

(iv) subadditivity: $\rho(X+Y) \leq \rho(X)+\rho(Y)$ for all $X, Y \in \mathcal{X}$.

(v) positive homogeneity: $\rho(\lambda X)=\lambda \rho(X)$ for all $X \in \mathcal{X}$ and $\lambda \in[0, \infty)$.

(vi) comonotonicity: $\rho(X+Y)=\rho(X)+\rho(Y)$ for all comonotone $X, Y \in \mathcal{X}$.

(vii) comonotonic convexity: $\rho(\lambda X+(1-\lambda) Y) \leq \lambda \rho(X)+(1-\lambda) \rho(Y)$ for all comonotone $X, Y \in \mathcal{X}$ and $\lambda \in[0,1]$. 
(viii) comonotonic subadditivity: $\rho(X+Y) \leq \rho(X)+\rho(Y)$ for all comonotonic $X, Y \in \mathcal{X}$.

(ix) law invariance: $\rho(X)=\rho(Y)$ for all $X, Y \in \mathcal{X}$ such that $X \sim Y$.

(x) surplus invariance subject to positivity: $\rho(X)=\rho\left(X^{+}\right)$for all $X \in \mathcal{X}$ such that $\rho(X) \geq 0$.

(xi) p-tail property: $\rho(X)=\rho(Y)$ for all $X, Y \in \mathcal{X}$ with $\operatorname{VaR}_{q}(X)=\operatorname{VaR}_{q}(Y)$ for all $q \in[p, 1)$.

Remark 4.1. Comonotonic subadditivity and convexity have been discussed in Song and Yan (2009). The property of surplus invariance subject to positivity was introduced in Koch-Medina et al. (2015) as a generalization of the loss-based property in Cont et al. (2013) and the excess invariance property in Staum (2013) and stipulates that capital requirements for unacceptable positions depend only on the loss tail of the distribution and are not affected by the shape of the surplus tail. Definition (xi) provides an equivalent formulation of the original tail property introduced in Liu and Wang (2016) that highlights the connection with VaR. We refer to Föllmer and Schied (2016) for a comprehensive presentation of the other standard properties.

Remark 4.2. It is well-known that VaR satisfies all the above properties but convexity and subadditivity. On the other side, ES satisfies all the above properties but surplus invariance subject to positivity.

Proposition 4.3. For every BLD $\alpha$ the risk measure $\mathrm{LVaR}_{\alpha}$ is monotone, stochastically monotone, comonotonically convex, law-invariant, surplus-invariant subject to positivity, and is a $p$-tail risk measure for all $p \in(0, \underline{\alpha}]$.

Proof. Monotonicity, stochastic monotonicity and law-invariance immediately follow from the corresponding properties of $\mathrm{VaR}$ in view of Proposition 3.7. To establish comonotonic convexity, take two comonotone $X, Y \in \mathcal{X}$ and $\lambda \in[0,1]$. Recalling that VaR is comonotonic and positive homogeneous, we use again Proposition 3.7 to obtain

$$
\begin{aligned}
\operatorname{LVaR}_{\alpha}(\lambda X+(1-\lambda) Y) & =\sup _{u \geq 0}\left(\operatorname{VaR}_{\alpha(u)}(\lambda X+(1-\lambda) Y)-u\right) \\
& =\sup _{u \geq 0}\left(\lambda \operatorname{VaR}_{\alpha(u)}(X)+(1-\lambda) \operatorname{VaR}_{\alpha(u)}(Y)-\lambda u-(1-\lambda) u\right) \\
& \leq \lambda \sup _{u \geq 0}\left(\operatorname{VaR}_{\alpha(u)}(X)-u\right)+(1-\lambda) \sup _{u \geq 0}\left(\operatorname{VaR}_{\alpha(u)}(Y)-u\right) \\
& =\lambda \operatorname{LVaR}_{\alpha}(X)+(1-\lambda) \operatorname{LVaR}_{\alpha}(Y) .
\end{aligned}
$$

To prove surplus-invariance subject to positivity, assume that $X \in \mathcal{A}_{\alpha}$ and take any $Y \in \mathcal{X}$ satisfying $Y^{+}=X^{+}$. Then, we easily see that

$$
F_{Y}(u)=F_{Y^{+}}(u)=F_{X^{+}}(u)=F_{X}(u) \geq \alpha(u)
$$

for all $u \geq 0$. This yields $Y \in \mathcal{A}_{\alpha}$. As a result, it follows from the definition of $\mathrm{LVaR}_{\alpha}$ that $\mathrm{LVaR}_{\alpha}$ is surplus-invariant subject to positivity. Finally, take an arbitrary $p \in(0, \underline{\alpha}]$ and assume that $X \in \mathcal{A}_{\alpha}$. Moreover, take any $Y \in \mathcal{X}$ satisfying $\operatorname{VaR}_{q}(Y)=\operatorname{VaR}_{q}(X)$ for all $q \in[p, 1)$. In particular, note that we have $F_{Y}(u)=F_{X}(u)$ for every $u \in\left[\operatorname{VaR}_{p}(X), \infty\right)$. This interval contains all positive real numbers because $\operatorname{VaR}_{p}(X) \leq \operatorname{VaR}_{\underline{\alpha}}(X) \leq \operatorname{LVaR}_{\alpha}(X) \leq 0$. Hence, it follows that

$$
F_{Y}(u)=F_{X}(u) \geq \alpha(u)
$$

for all $u \geq 0$, which yields $Y \in \mathcal{A}_{\alpha}$. As a result, we can apply Proposition 3.1 in Liu and Wang (2016) to conclude that $\mathrm{LVaR}_{\alpha}$ is a $p$-tail risk measure. 
Remark 4.4. It follows from Proposition 3.3 that every function $\rho: \mathcal{X} \rightarrow[-\infty, \infty]$ that is monotonic, stochastically monotonic, cash-additive, and satisfies $\rho(0)=0$ can be expressed as

$$
\rho(X)=\inf _{\alpha \in D} \operatorname{LVaR}_{\alpha}(X)
$$

for every $X \in \mathcal{X}$, where $D$ is a suitable class of BLDs. To see this, set $\mathcal{A}=\{X \in \mathcal{X} ; \rho(X) \leq 0\}$ as well as $\mathcal{A}_{Y}=\left\{X \in \mathcal{X} ; X \leq_{s t} Y\right\}$ for every $Y \in \mathcal{X}$ and note that

$$
\mathcal{A}=\bigcup_{Y \in \mathcal{A}} \mathcal{A}_{Y}
$$

The inclusion " $\supset$ " follows from stochastic monotonicity. Then, we easily have

$$
\rho(X)=\rho_{\mathcal{A}}(X)=\inf _{Y \in \mathcal{A}} \rho_{\mathcal{A}_{Y}}(X)=\inf _{Y \in \mathcal{A}, \varepsilon>0} \rho_{\mathcal{A}_{Y-\varepsilon}}(X)
$$

for every $X \in \mathcal{X}$. Note that, for every $Y \in \mathcal{A}$ and every $\varepsilon>0$, we have $Y-\varepsilon \in \mathcal{A}$ and $F_{Y-\varepsilon}(0)>0$ (otherwise $Y \geq \varepsilon$ and, thus, $\rho(Y) \geq \rho(\varepsilon)=\varepsilon$ by monotonicity and cash-additivity). The desired representation follows from Proposition 3.3 by setting $D=\left\{\alpha_{Y, \varepsilon} ; Y \in \mathcal{A}, \varepsilon>0\right\}$, where $\alpha_{Y, \varepsilon}(u)=F_{Y-\varepsilon}(u)$ for $u \geq 0$. The above representation can be viewed as a first-order dominance counterpart of the representation of consistent risk measures obtained in Theorem 3.1 in Mao and Wang (2016). We thank Ruodu Wang for pointing this out.

The next proposition shows how the capital requirements for the multiple of a random position can be controlled by the original capital requirements. In particular, it shows that if we increase the size of a position, the capital requirement for the rescaled position is higher than the rescaled capital requirement.

Proposition 4.5. For every $B L D \alpha$ and all $X \in \mathcal{X}$ the risk measure $\operatorname{LVaR}_{\alpha}$ satisfies:

(i) $\operatorname{LVaR}_{\alpha}(\lambda X) \leq \lambda \operatorname{LVaR}_{\alpha}(X)$ for all $0<\lambda<1$.

(ii) $\operatorname{LVaR}_{\alpha}(\lambda X) \geq \lambda \operatorname{LVaR}_{\alpha}(X)$ for all $\lambda>1$.

Proof. It is enough to prove assertion (i). To this effect, take arbitrary $X \in \mathcal{X}$ and $0<\lambda<1$ and note that

$$
\operatorname{LVaR}_{\alpha}(\lambda X)=\sup _{u \geq 0}\left(\operatorname{VaR}_{\alpha(u)}(\lambda X)-u\right) \leq \sup _{u \geq 0}\left(\lambda \operatorname{VaR}_{\alpha(u)}(X)-\lambda u\right)=\lambda \operatorname{LVaR}_{\alpha}(X)
$$

by positive homogeneity of VaR. This proves assertion (i).

The next proposition shows that LVaR fails to be convex or subadditive unless it coincides with the worst-scenario risk measure. In addition, LVaR also fails to be positively homogeneous, comonotonic and comonotonic subadditive unless it reduces to VaR.

Proposition 4.6. For every BLD $\alpha$ the following statements hold:

(i) The following assertions are equivalent:

(a) $\mathrm{LVaR}_{\alpha}$ is convex.

(b) $\mathrm{LVaR}_{\alpha}$ is subadditive.

(c) $\alpha$ is constant with $\underline{\alpha}=1$. 
In this case, $\operatorname{LVaR}_{\alpha}(X)=\operatorname{ess} \sup (X)$ for all $X \in \mathcal{X}$.

(ii) The following assertions are equivalent:

(a) $\mathrm{LVaR}_{\alpha}$ is positively homogeneous.

(b) $\mathrm{LVaR}_{\alpha}$ is comonotonic.

(c) $\mathrm{LVaR}_{\alpha}$ is comonotonically subadditive.

(d) $\alpha$ is constant.

In this case, $\operatorname{LVaR}_{\alpha}(X)=\operatorname{VaR}_{\underline{\alpha}}(X)$ for all $X \in \mathcal{X}$.

Proof. (i) It is clear that (c) implies (b), which implies (a) by virtue of point (iii). To prove that (a) implies (c), assume that $\underline{\alpha}<1$ and take an arbitrary

$$
0<\varepsilon<\min \left(\frac{1-\underline{\alpha}}{3}, \underline{\alpha}\right) .
$$

Note that, by nonatomicity, we can always find suitable events $E, F \in \mathcal{F}$ such that

$$
\mathbb{P}(E)=\mathbb{P}(F)=\underline{\alpha}+\varepsilon \quad \text { and } \quad \mathbb{P}(E \cap F)=\underline{\alpha}-\varepsilon .
$$

Moreover, since $\alpha$ is right-continuous, there exists $z>0$ satisfying $\alpha(z)<\underline{\alpha}+\varepsilon$. Then, it is easy to see that the random variables $X=z \mathbf{1}_{E^{c}}$ and $Y=z \mathbf{1}_{F^{c}}$ satisfy

$$
F_{X}(u)=F_{Y}(u)= \begin{cases}\underline{\alpha}+\varepsilon>\alpha(z) \geq \alpha(u) & \text { if } 0 \leq u<z, \\ 1 \geq \alpha(u) & \text { if } u \geq z .\end{cases}
$$

This shows that both $X$ and $Y$ belong to $\mathcal{A}_{\alpha}$. However, we have $F_{\frac{1}{2} X+\frac{1}{2} Y}(0)=\mathbb{P}(E \cap F)=$ $\underline{\alpha}-\varepsilon<\underline{\alpha}$, proving that $\frac{1}{2} X+\frac{1}{2} Y$ lies outside $\mathcal{A}_{\alpha}$. As a result, it follows that

$$
\frac{1}{2} \operatorname{LVaR}_{\alpha}(X)+\frac{1}{2} \operatorname{LVaR}_{\alpha}(Y) \leq 0<\operatorname{LVaR}_{\alpha}\left(\frac{1}{2} X+\frac{1}{2} Y\right),
$$

showing that $\mathrm{LVaR}_{\alpha}$ is not convex. Hence, (a) implies that $\underline{\alpha}=1$. In this case, $\alpha$ must be constant.

(ii) Note that $\operatorname{LVaR}_{\alpha}$ coincides with $\operatorname{VaR}_{\underline{\alpha}}$ whenever $\alpha$ is constant. Hence, (d) implies any of the other assertions. To establish the converse implication, assume that $\alpha$ is not constant so that $\alpha(y)<\alpha(z)$ for some $0<y<z$ and consider a random variable $X \in \mathcal{X}$ satisfying

$$
F_{X}(u)= \begin{cases}0 & \text { if } u<0 \\ p & \text { if } 0 \leq u<x \\ 1 & \text { if } u \geq x\end{cases}
$$

for some $p \in(\alpha(y), \alpha(z))$ and $x>z$. Note that $0<y \leq \alpha_{+}^{-1}(p) \leq z<x$. Then, we have

$\operatorname{LVaR}_{\alpha}(X)=\max \left(0, x-\alpha_{+}^{-1}(p)\right)=x-\alpha_{+}^{-1}(p)$ and $\operatorname{LVaR}_{\alpha}(2 X)=\max \left(0,2 x-\alpha_{+}^{-1}(p)\right)=2 x-\alpha_{+}^{-1}(p)$

by Proposition 3.11. Since $\alpha_{+}^{-1}(p)>0$, we easily see that $\operatorname{LVaR}_{\alpha}(2 X)>2 \operatorname{LVaR}_{\alpha}(X)$, which shows that $\mathrm{LVaR}_{\alpha}$ is not positively homogeneous, neither comonotonic nor comonotonically subadditive. 
Remark 4.7. The lack of subadditivity implies that LVaR exhibits regulatory arbitrage as introduced by Wang (2016). In the presence of regulatory arbitrage a financial institution can reduce capital requirements, at least at a theoretical level, by splitting its portfolio into several subportolios. As shown in Proposition 2.1 in Wang (2016) a risk measure is free of regulatory arbitrage if and only if it is subadditive. For non-subadditive risk measures the question is whether the arbitrage is limited or not, meaning that, when the number of subportfolios goes to infinity, the reduction of capital requirement is bounded or not. For the case of a benchmark loss distribution with upper bound $\bar{\alpha}<1$, i.e. for the case where LVaR is bounded from above by some VaR, it follows from Proposition 2.6 in Wang (2016) that the regulatory arbitrage is unlimited. For the case $\bar{\alpha}=1$ the answer is, a priori, unclear. Note, however, that the regulatory arbitrage can always be limited by simply taking the maximum between LVaR and, for example, the mean; see Corollary 5.1 in Wang (2016). This is a mild restriction since, typically, regulators will not enforce capital rule which are less conservative than the mean.

We conclude this section by highlighting that LVaR somewhat "inherits" convexity from VaR when applied to combinations of random variables for which VaR is convex. Condition (4) below is satisfied, for instance, whenever $X$ and $Y$ have a joint elliptical distribution and $\underline{\alpha} \geq \frac{1}{2}$.

Proposition 4.8. Take $X, Y \in \mathcal{X}$ and assume that for all $p \in[\underline{\alpha}, 1)$ and $\lambda \in[0,1]$ we have

$$
\operatorname{VaR}_{p}(\lambda X+(1-\lambda) Y) \leq \lambda \operatorname{VaR}_{p}(X)+(1-\lambda) \operatorname{VaR}_{p}(Y) .
$$

Then, for all $\lambda \in[0,1]$ we have

$$
\operatorname{LVaR}_{\alpha}(\lambda X+(1-\lambda) Y) \leq \lambda \operatorname{LVaR}_{\alpha}(X)+(1-\lambda) \operatorname{LVaR}_{\alpha}(Y) .
$$

Proof. For every $\lambda \in[0,1]$ it follows from Proposition 3.7 that

$$
\begin{aligned}
\operatorname{LVaR}_{\alpha}(\lambda X+(1-\lambda) Y) & =\sup _{u \geq 0}\left(\operatorname{VaR}_{\alpha(u)}(\lambda X+(1-\lambda) Y)-\lambda u-(1-\lambda) u\right) \\
& \leq \sup _{u \geq 0}\left(\lambda\left(\operatorname{VaR}_{\alpha(u)}(X)-u\right)+(1-\lambda)\left(\operatorname{VaR}_{\alpha(u)}(Y)-u\right)\right) \\
& \leq \lambda \operatorname{LVaR}_{\alpha}(X)+(1-\lambda) \operatorname{LVaR}_{\alpha}(Y) .
\end{aligned}
$$

This concludes the proof of the proposition.

\section{$5 \quad$ Statistical properties}

As mentioned in the introduction, the recent literature has devoted much attention to investigating the statistical properties of VaR and ES and of general risk measures. The main goal of this new strand of research is to assess the statistical reliability of the risk measurement procedure associated to a given risk metric. As described by Cont et al. (2010), the risk measurement procedure consists of two fundamental steps. First, the distribution of some financial position has to be estimated from a set of available data such as historical observations or simulations. Second, the estimated distribution is used to compute a capital requirement or a risk indicator by applying a pre-specified (law-invariant) risk measure. In this section we focus on the risk measurement procedure involving LVaR with a special focus on robustness, elicitability, and backtestability.

As is customary in the statistical literature, we will freely switch from a law-invariant risk measure $\rho: \mathcal{X} \rightarrow[-\infty,+\infty]$ to the corresponding statistical functional $\rho: \mathcal{P} \rightarrow[-\infty,+\infty]$ (we use the same symbol by abuse of notation) defined by

$$
\rho(F):=\rho\left(X_{F}\right)
$$


where $\mathcal{P}$ is the set of probability distribution functions on $\mathbb{R}$ and $X_{F}$ denotes any random variable with distribution function $F$.

\section{Robustness}

The robustness of the outcome of a risk measurement procedure with respect to perturbations in the data set and with respect to different estimation procedures for the underlying distribution is highly desirable from an operational perspective. In this paper we focus on statistical robustness in the sense of Cont et al. (2010). We refer to Krätschmer et al. (2014) and to the survey paper Embrechts et al. (2014) for a discussion on alternative notions of robustness.

In what follows we denote by $d_{L}(F, G)$ the Lévy distance between $F, G \in \mathcal{P}$, i.e.

$$
d_{L}(F, G):=\inf \{\varepsilon>0 ; G(x-\varepsilon)-\varepsilon \leq F(x) \leq G(x+\varepsilon)+\varepsilon, \forall x \in \mathbb{R}\} .
$$

To each statistical functional $\rho: \mathcal{P} \rightarrow[-\infty,+\infty]$ we associate a risk estimator

$$
\widehat{\rho}: \mathbb{X} \rightarrow \mathbb{R}
$$

where $\mathbb{X}:=\bigcup_{n \in \mathbb{N}} \mathbb{R}^{n}$. Every vector $\mathbf{x} \in \mathbb{X}$ is interpreted as a collection of data generated by some underlying distribution $F \in \mathcal{P}$ and the quantity $\widehat{\rho}(\mathbf{x})$ has to be understood as an estimate for $\rho(F)$. A typical example is the historical estimator defined by

$$
\widehat{\rho}_{h}(\mathbf{x}):=\rho\left(\frac{1}{n} \sum_{k=1}^{n} \mathbf{1}_{\left[x_{k}, \infty\right)}\right)
$$

for all $\mathbf{x} \in \mathbb{R}^{n}$. We refer to Cont et al. (2010) for more details on risk estimators. Provided that $\widehat{\rho}$ is measurable with respect to a suitable measurable structure on $\mathbb{X}$, assumption that we make throughout the section, we can define for any $F \in \mathcal{P}$ and $n \in \mathbb{N}$ the random variable

$$
\widehat{\rho}_{n}(F):=\widehat{\rho}\left(\mathbf{X}_{F}^{(n)}\right)
$$

where $\mathbf{X}_{F}^{(n)}$ is any $n$-dimensional vector of i.i.d. random variables with common distribution $F$.

Definition 5.1. Let $\rho: \mathcal{P} \rightarrow[-\infty,+\infty]$ be a statistical functional. We say that $\rho$ is continuous at $F \in \mathcal{P}$ if for every $\varepsilon>0$ there exists $\delta>0$ such that

$$
G \in \mathcal{P}, d_{L}(F, G)<\delta \Longrightarrow|\rho(F)-\rho(G)|<\varepsilon .
$$

Let $\mathcal{D} \subset \mathcal{P}$. We say that a risk estimator $\widehat{\rho}$ is $\mathcal{D}$-robust at $F \in \mathcal{P}$ if for every $\varepsilon>0$ there exist $\delta>0$ and $n_{0} \in \mathbb{N}$ such that

$$
G \in \mathcal{D}, d_{L}(F, G)<\delta \Longrightarrow \sup _{n \geq n_{0}} d_{L}\left(F_{\widehat{\rho}_{n}(F)}, F_{\widehat{\rho}_{n}(G)}\right)<\varepsilon
$$

Intuitively, if a risk estimator is robust, then a slight perturbation in a certain class of distributions $\mathcal{D}$ yields a small change in the estimated risk measure. The larger the set $\mathcal{D}$, the more the risk estimation will be stable under different types of perturbations (for $\mathcal{D}=\mathcal{P}$ one speaks of qualitative robustness, see Huber, 1981). The following result highlights the link between continuity and robustness.

Proposition 5.2 (Corollary 3.3 in Cont et al., 2010). Let $\mathcal{D} \subset \mathcal{P}$. For a statistical functional $\rho: \mathcal{P} \rightarrow[-\infty,+\infty]$ the following statements are equivalent: 
(a) $\rho$ is continuous on $\mathcal{D}$.

(b) $\widehat{\rho}_{h}$ is $\mathcal{D}$-robust on $\mathcal{D}$.

In line with the observation by Yamai and Yoshiba (2005), it was highlighted in Cont et al. (2010) that VaR requires less data than ES to be estimated accurately and is more robust than ES because it ignores any change in the data beyond the VaR threshold. As a tradeoff between the tail blindness of VaR and the lack of robustness of ES, Cont et al. (2010) introduced the new risk measure RVaR; see Section 2. Being an average of quantiles between two VaR thresholds, RVaR captures more tail information than VaR but, at the same time, does not require the estimation of the entire tail and is thus more robust than ES. A broad overview of RVaR has been recently provided in Embrechts et al. (2018).

We start by showing that LVaR is always robust on strictly increasing distribution functions provided that $\alpha$ is a stepwise benchmark function. This includes, of course, the case of a constant benchmark function. In what follows, we denote by $\mathcal{D}_{\uparrow}$ the set of strictly increasing distribution functions and by $\mathcal{D}_{\uparrow}^{c}$ its subset consisting of continuous functions.

Proposition 5.3. Consider a piecewise constant $B L D \alpha$ as in Proposition 3.10. Then, $\mathrm{LVaR}_{\alpha}$ is continuous on $\mathcal{D}_{\uparrow}$.

Proof. Take arbitrary $F, G \in \mathcal{D}_{\uparrow}$ and recall that

$$
\operatorname{LVaR}_{\alpha}(F)=\max _{k=1, \ldots, n}\left(\operatorname{VaR}_{\alpha_{k}}(F)-u_{k}\right)
$$

where $0<\alpha_{1}<\cdots<\alpha_{n} \leq 1$ and $0=u_{1}<\cdots<u_{n}$ are as in Proposition 3.10. Since $\operatorname{VaR}_{p}$

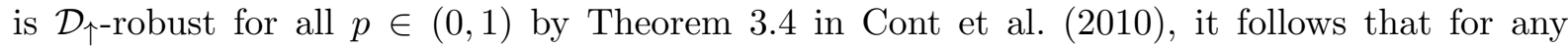
$k \in\{1, \ldots, n\}$ and for any $\varepsilon>0$ there exists $\delta_{k}>0$ such that $\left|\operatorname{VaR}_{\alpha_{k}}(F)-\operatorname{VaR}_{\alpha_{k}}(G)\right|<\varepsilon$ whenever $d_{L}(F, G)<\delta_{k}$. Setting $\delta=\min \left(\delta_{1}, \ldots, \delta_{n}\right)$ we see that

$$
\operatorname{VaR}_{\alpha_{k}}(G)-u_{k}-\varepsilon \leq \operatorname{VaR}_{\alpha_{k}}(F)-u_{k} \leq \operatorname{VaR}_{\alpha_{k}}(G)-u_{k}+\varepsilon
$$

for all $k \in\{1, \ldots, n\}$ provided that $d_{L}(F, G)<\delta$. By taking the maximum over $k$ we conclude that $d_{L}(F, G)<\delta$ implies $\left|\operatorname{LVaR}_{\alpha}(F)-\operatorname{LVaR}_{\alpha}(G)\right|<\varepsilon$, proving continuity on $\mathcal{D}_{\uparrow}$.

If the benchmark function is continuous, then robustness holds on strictly increasing distribution functions that are additionally assumed to be continuous. Before proving this, we need the following preliminary result.

Lemma 5.4. Let $F \in \mathcal{D}_{\uparrow}^{c}$ and let $\alpha$ be a continuous BLD. Then, the following statements hold:

(a) If $\bar{\alpha}<1$, then for every $\varepsilon>0$ there exists $\xi \in(0,1-\bar{\alpha})$ such that

$$
\sup _{u \geq 0}\left(\operatorname{VaR}_{\alpha(u)+\xi}(F)-\operatorname{VaR}_{\alpha(u)}(F)\right) \leq \varepsilon .
$$

(b) For every $\varepsilon>0$ there exists $\xi \in(0, \underline{\alpha})$ such that

$$
\sup _{u \geq 0}\left(\operatorname{VaR}_{\alpha(u)}(F)-\operatorname{VaR}_{\alpha(u)-\xi}(F)\right) \leq \varepsilon .
$$


Proof. Take $\varepsilon>0$ and for each $u \geq 0$ define $\xi(u)=F\left(F^{-1}(\alpha(u))+\varepsilon\right)-\alpha(u)$. Moreover, set

$$
\xi=\min \left(\inf _{u \geq 0} \xi(u), \frac{1-\bar{\alpha}}{2}\right) .
$$

Note that $\xi(u)>0$ since $F$ is strictly increasing and that $\lim _{u \rightarrow \infty} \xi(u)>0$ because $\bar{\alpha}<1$. As a result of the continuity of $F$ and $\alpha$, we infer that $\xi>0$. To conclude the proof of the first assertion it suffices to observe that

$$
\operatorname{VaR}_{\alpha(u)+\xi}(F) \leq \operatorname{VaR}_{\alpha(u)+\xi(u)}(F)=F^{-1}(\alpha(u)+\xi(u))=F^{-1}(\alpha(u))+\varepsilon=\operatorname{VaR}_{\alpha(u)}(F)+\varepsilon
$$

for all $u \geq 0$. This establishes (a). The proof of (b) is analogous once we recall that $\underline{\alpha}>0$.

Proposition 5.5. Consider a continuous BLD $\alpha$ such that $\bar{\alpha}<1$. Then, $\mathrm{LVaR}_{\alpha}$ is continuous on $\mathcal{D}_{\uparrow}^{c}$.

Proof. Fix $F \in \mathcal{P}$ and $\varepsilon>0$ and take $\xi \in(0,1-\bar{\alpha})$ such that

$$
\sup _{u \geq 0}\left(\operatorname{VaR}_{\alpha(u)+\xi}(F)-\operatorname{VaR}_{\alpha(u)}(F)\right) \leq \varepsilon .
$$

This is possible by point (a) in Lemma 5.4. Now, take $\delta \in(0, \min \{\xi, \varepsilon\})$ and let $G \in \mathcal{P}$ satisfy $d_{L}(F, G)<\delta$. By definition of Lévy distance, for all $m \in \mathbb{R}$ and $u \geq 0$ we have

$$
F(u+m)-\delta \geq \alpha(u) \Longrightarrow G(u+m+\delta) \geq \alpha(u) .
$$

This yields $\operatorname{LVaR}_{\alpha}(G) \leq \rho_{\alpha+\delta}(F)+\delta$. As a result, we infer from (5) that

$$
\begin{aligned}
\operatorname{LVaR}_{\alpha}(G) & \leq \sup _{u \geq 0}\left(\operatorname{VaR}_{\alpha(u)+\delta}(F)-u\right)+\varepsilon \\
& \leq \sup _{u \geq 0}\left(\operatorname{VaR}_{\alpha(u)+\xi}(F)-u\right)+\varepsilon \\
& \leq \sup _{u \geq 0}\left(\operatorname{VaR}_{\alpha(u)}(F)-u\right)+2 \varepsilon \\
& =\operatorname{LVaR}_{\alpha}(F)+2 \varepsilon .
\end{aligned}
$$

In a similar way, we can establish that $\operatorname{LVaR}_{\alpha}(G) \geq \operatorname{LVaR}_{\alpha}(F)-2 \varepsilon$ by using point (b) in Lemma 5.4. This shows that $\mathrm{LVaR}_{\alpha}$ is continuous at $F$ and concludes the proof.

A simple example shows that robustness of LVaR fails, in general, if we do not assume that the benchmark loss distribution is bounded away from 1. This is in line with the findings of Cont et al. (2010), where robustness was shown to fail for risk measures taking into account the full spectrum of quantiles. It is also known that VaR is robust only on the set of strictly increasing distribution functions (more precisely, $\mathrm{VaR}_{p}$ is robust, for any $p \in(0,1)$, on the set of distributions having a unique $p$-quantile). Therefore, also the restriction to the class $\mathcal{D}_{\uparrow}$ is required.

Example 5.6. Let $\gamma>1$ and consider the functions

$$
F(x)=\left\{\begin{array}{ll}
\frac{1}{2} e^{\gamma x} & \text { if } x<0, \\
1-\frac{1}{2} e^{-\gamma x} & \text { if } x \geq 0,
\end{array} \quad \text { and } \quad \alpha(u)=F(u), \quad u \geq 0 .\right.
$$


Since $F$ and $\alpha$ coincides on $[0, \infty)$ and $F$ is strictly increasing, we clearly have $\operatorname{LVaR}_{\alpha}(F)=0$. Take now an arbitrary $\delta \in(0,1)$. We show that there exists a distribution function $G$ satisfying $d_{L}(F, G) \leq \delta$ and $\operatorname{LVaR}_{\alpha}(G)=\infty$. To this effect, define

$$
G(x)= \begin{cases}F(x)-\frac{\delta}{2} e^{(\gamma-1) x} & \text { if } x<0 \\ F(x)-\frac{\delta}{2} e^{-(\gamma-1) x} & \text { if } x \geq 0\end{cases}
$$

It can be easily checked that $G$ is a distribution function. Moreover, $G(x) \geq F(x)-\delta \geq$ $F(x-\delta)-\delta$ and $G(x) \leq F(x) \leq F(x+\delta)+\delta$ for all $x \in \mathbb{R}$. Thus, $d_{L}(F, G) \leq \delta$ holds. Fix now $m \in \mathbb{R}$ and note that, for any $x \geq \max (0,-m)$, we have

$$
\begin{aligned}
G(x+m) \geq \alpha(x) & \Longleftrightarrow 1-\frac{1}{2} e^{-\gamma(x+m)}-\frac{\delta}{2} e^{-(\gamma-1)(x+m)} \geq 1-\frac{1}{2} e^{-\gamma x} \\
& \Longleftrightarrow e^{-\gamma(x+m)}+\delta e^{-(\gamma-1)(x+m)} \leq e^{-\gamma x} \\
& \Longleftrightarrow e^{-\gamma(x+m)}\left(1+\delta e^{x+m}-e^{\gamma m}\right) \leq 0 \\
& \Longleftrightarrow 1+\delta e^{x+m}-e^{\gamma m} \leq 0 .
\end{aligned}
$$

Since the above inequality cannot hold for $x$ large enough, we conclude that $\operatorname{LVaR}_{\alpha}(G)=\infty$.

We conclude the section on robustness by highlighting that LVaR is "robust" with respect to changes in the underlying benchmark loss distribution. This is a direct consequence of Lemma 5.4 .

Proposition 5.7. Let $F \in \mathcal{D}_{\uparrow}^{c}$ and consider two BLDs $\alpha$ and $\beta$ with $\bar{\alpha}<1$ and $\bar{\beta}<1$. Then, for every $\varepsilon>0$ there exists $\delta>0$ such that

$$
\sup _{u \geq 0}|\alpha(u)-\beta(u)|<\delta \Longrightarrow\left|\operatorname{LVaR}_{\alpha}(F)-\operatorname{LVaR}_{\beta}(F)\right|<\varepsilon .
$$

\section{Backtestability}

As mentioned in the introduction, backtestability is another important statistical property of risk measures. Roughly speaking, a risk measure is backtestable if it allows to test, a posteriori, the accuracy of the estimation procedure involved in the risk measurement process. The notion of backtestability is close to that of elicitability, which has been the subject of an intense academic debate in the recent years. After recalling the notion of elicitability, we show that risk measures based on benchmark loss distributions are not elicitable in general. Nevertheless, we discuss some possible procedures for performing backtesting.

Definition 5.8. A statistical functional $\rho: \mathcal{P} \rightarrow[-\infty,+\infty]$ is elicitable if there exists a measurable function $L: \mathbb{R} \times \mathbb{R} \rightarrow[0, \infty)$ such that for every $F \in \mathcal{P}$ we have

$$
\rho(F)=\underset{z \in \mathbb{R}}{\arg \min } \int_{\mathbb{R}} L(z, x) \mathrm{d} F(x) .
$$

Under weak assumptions the "loss function" $L$ allows to rank different risk measure estimates based on their empirical average loss; see for instance Bellini and Bignozzi (2015) and the references therein. In addition, as discussed in Bellini et al. (2018), the function $L$ can be employed to test whether a single estimate for $\rho(F)$ is sufficiently accurate in the sense that it is close to minimize the empirical expected loss. As pointed out by Gneiting (2011), elicitability is satisfied by VaR (on the set of strictly increasing distributions) but not by ES, which is only jointly elicitable with VaR (see Fissler et al. (2016) and the references therein). We refer to 
Bellini and Bignozzi (2015), Ziegel (2016), Acerbi and Szekely (2017), Bellini and Di Bernardino (2017), and Nolde and Ziegel (2017) for more details on elicitability and backtesting.

A necessary condition for elicitability, see e.g. Delbaen et al. (2016), is the Convex Level Sets (CxLS) property requiring that

$$
\rho(\lambda F+(1-\lambda) G)=\rho(F)=\rho(G)
$$

for all $\lambda \in[0,1]$ and $F, G \in \mathcal{P}$ such that $\rho(F)=\rho(G)$. It is well-known that VaR satisfies CxLS. Hence, $\operatorname{LVaR}_{\alpha}$ satisfies CxLS for all distributions $F, G \in \mathcal{P}$ such that $\operatorname{VaR}_{\alpha(u)}(F)=\operatorname{VaR}_{\alpha(u)}(G)$ for every $u \geq 0$. However, $\mathrm{LVaR}_{\alpha}$ fails to satisfy $\mathrm{CxLS}$ for general distributions and, thus, is not elicitable.

Example 5.9. Consider $0<\alpha_{1}<\alpha_{2}<1$ such that $2 \alpha_{2}-\alpha_{1}<1$ and define the functions

$$
F(x)=\left\{\begin{array}{ll}
\left(2 \alpha_{1}-\alpha_{2}\right) e^{x} & \text { if } x<0, \\
\left(\alpha_{2}-\alpha_{1}\right) x+2 \alpha_{1}-\alpha_{2} & \text { if } 0 \leq x<3, \\
1-\left(1-2 \alpha_{2}+\alpha_{1}\right) e^{x-3} & \text { if } x \geq 3,
\end{array} \quad G(x)= \begin{cases}\alpha_{1} e^{x} & \text { if } x<0, \\
\frac{\alpha_{2}-\alpha_{1}}{3} x+\alpha_{1} & \text { if } 0 \leq x<3 \\
1-\left(1-\alpha_{2}\right) e^{x-3} & \text { if } x \geq 3 .\end{cases}\right.
$$

Then, consider the benchmark loss distribution

$$
\alpha(u)= \begin{cases}\alpha_{1} & \text { if } 0 \leq u<2 \\ \alpha_{2} & \text { if } u \geq 2\end{cases}
$$

Note that $F(u)<\alpha_{1}$ for all $0 \leq u<1$ as well as $F(1)=\alpha_{1}$ and $F(2)=\alpha_{2}$. Hence, we have $\operatorname{LVaR}_{\alpha}(F)=1$. Similarly, $G(u)<\alpha_{2}$ for all $2 \leq u<3$ as well as $G(0)=\alpha_{1}$ and $G(3)=\alpha_{2}$. This yields $\operatorname{LVaR}_{\alpha}(G)=1$. However, it is easy to verify that

$$
\operatorname{LVaR}_{\alpha}(\lambda F+(1-\lambda) G)=\max \left(\frac{3 \lambda}{2 \lambda+1}, \frac{1-\lambda}{2 \lambda+1}\right)<1
$$

for all $\lambda \in(0,1)$, showing that $\mathrm{LVaR}_{\alpha}$ does not satisfy CxLS.

In the remainder we focus on the following definition of backtestability, which has been recently proposed by Acerbi and Szekely (2017).

Definition 5.10. Let $\mathcal{D} \subset \mathcal{P}$. A statistical functional $\rho: \mathcal{P} \rightarrow[-\infty,+\infty]$ is $\mathcal{D}$-identifiable if there exists a measurable function $B: \mathbb{R} \times \mathbb{R} \rightarrow \mathbb{R}$ such that

$$
\int_{\mathbb{R}} B(z, x) \mathrm{d} F(x)=0 \Longleftrightarrow z=\rho(F)
$$

for all $F \in \mathcal{D}$. We say that $\rho$ is $\mathcal{D}$-backtestable if, in addition, $B$ satisfies

$$
z_{1}<z_{2} \Longrightarrow \int_{\mathbb{R}} B\left(z_{1}, x\right) \mathrm{d} F(x)<\int_{\mathbb{R}} B\left(z_{2}, x\right) \mathrm{d} F(x)
$$

for all $F \in \mathcal{D}$.

Our discussion on backtestability is inspired by Acerbi and Szekely (2017), although a different analysis is required for our case. Indeed, LVaR cannot be backtestable in the above sense because backtestability implies elicitability by Proposition 3.8 in Acerbi and Szekely (2017) and we know that LVaR is not elicitable in general. As a preliminary observation, recall from Proposition 3.2 
in Acerbi and Szekely (2017) that, for every $p \in(0,1), \operatorname{VaR}_{p}$ is $\mathcal{D}_{\uparrow}^{c}$-backtestable with backtesting function $B_{p}: \mathbb{R} \times \mathbb{R} \rightarrow \mathbb{R}$ given by

$$
B_{p}(z, x):= \begin{cases}1-p & \text { if } z \geq x \\ -p & \text { otherwise }\end{cases}
$$

In particular, note that $\int_{\mathbb{R}} B_{p}(z, x) \mathrm{d} F(x)=F(z)-p$ for all $z \in \mathbb{R}$ and $F \in \mathcal{P}$.

Now, suppose that $z \in \mathbb{R}$ is an estimation of $\operatorname{LVaR}_{\alpha}(F)$ for a given distribution $F \in \mathcal{D}_{\uparrow}^{c}$. We know from Proposition 3.7 that $\operatorname{LVaR}_{\alpha}(F)+u \geq \operatorname{VaR}_{\alpha(u)}(F)$ must hold for all $u \geq 0$. As a result, the backtestability of $\mathrm{VaR}$ yields

$$
\inf _{u \geq 0} \int_{\mathbb{R}} B_{\alpha(u)}\left(\operatorname{LVaR}_{\alpha}(F)+u, x\right) \mathrm{d} F(x) \geq 0 .
$$

This implies that $z$ does not underestimate $\operatorname{LVaR}_{\alpha}(F)$ provided that

$$
\inf _{u \geq 0} \int_{\mathbb{R}} B_{\alpha(u)}(z+u, x) \mathrm{d} F(x) \geq 0 .
$$

Even though we cannot "identify" $\operatorname{LVaR}_{\alpha}(F)$ in the sense of Definition 5.10, the above test allows us to infer whether we are (grossly) underestimating risk or not. In the common situation where the supremum in Proposition 3.7 is attained at some $u^{*} \geq 0$, as happens if $\alpha$ takes finitely many values, then $\operatorname{LVaR}_{\alpha}(F)+u^{*}=\operatorname{VaR}_{\alpha\left(u^{*}\right)}(F)$ so that

$$
\inf _{u \geq 0} \int_{\mathbb{R}} B_{\alpha(u)}\left(\operatorname{LVaR}_{\alpha}(F)+u, x\right) \mathrm{d} F(x)=0 .
$$

In this case, we expect a good estimate $z$ for $\operatorname{LVaR}_{\alpha}(F)$ to satisfy

$$
\inf _{u \geq 0} \int B_{\alpha(u)}(z+u, x) \mathrm{d} F(x)=0 .
$$

The above considerations suggest different options for both model selection and model validation based on LVaR. Following Acerbi and Szekely (2017), we use the term model selection to refer to the choice of the best risk measurement procedure among a class of competitive methods, e.g. based on empirical distributions or parametric distributions estimated via maximum likelihood. It is worth stressing that model selection provides a comparison among different estimation procedures but does not specify their accuracy in terms of the proximity to the true value $\operatorname{LVaR}_{\alpha}(F)$. On the other hand, model validation refers to testing the accuracy of a specific estimation procedure with respect to the true value of the risk measure.

In what follows we denote by $\mathbf{x} \in \mathbb{R}^{n}$ a sequence of realizations of i.i.d. random variables with common distribution $F \in \mathcal{D}_{\uparrow}^{c}$.

Model selection. Suppose we first want to select among $M$ different estimations of $\operatorname{LVaR}_{\alpha}(F)$ denoted by $z^{(1)}, \ldots, z^{(M)} \in \mathbb{R}$ and produced using distribution functions $F^{(1)}, \ldots, F^{(M)} \in \mathcal{D}_{\uparrow}^{c}$. For every $u \geq 0$ we introduce the realized mean

$$
\mu_{u}^{(k)}=\frac{1}{n} \sum_{j=1}^{n} B_{\alpha(u)}\left(z^{(k)}+u, x_{j}\right)
$$

for $k=1, \ldots, M$. If the estimation $z^{(k)}$ coincides with the exact value $\operatorname{LVaR}_{\alpha}(F)$, then $\mu_{u}^{(k)}$ must be positive for all $u \geq 0$ and close to zero for every $u$ close to

$$
\bar{u}^{(k)}=\underset{u \geq 0}{\arg \max }\left(\operatorname{VaR}_{\alpha(u)}\left(F^{(k)}\right)-u\right) .
$$

The model selection procedure can be performed in different ways: 
(a) Compute the realized mean $\mu_{u}^{(k)}$ only for the minimizer $u=\bar{u}^{(K)}$ and choose the estimation with the lowest rank.

(b) Compute the realized mean $\mu_{u}^{(k)}$ for a collection of $u$ close to $\bar{u}^{(K)}$ : The best estimation should have realized mean close to zero for any of those $u$.

(c) Compute the realized mean $\mu_{u}^{(k)}$ for a large collection of $u$ : The best estimation should have non-negative realized mean for all $u$ and be close to zero for $u=\bar{u}^{(K)}$.

Model validation. Along the same lines one can propose different methods for model validation. Of course, the procedures described above can be also applied in a model validation setting. In addition, we may exploit the remarkable fact that VaR does not require any condition on the data generating process to allow for model validation in order to describe another model validation procedure for LVaR. The key observation is that, for every $z \in \mathbb{R}$ and every random variable $X_{F}$ with distribution $F \in \mathcal{D}_{\uparrow}^{c}$ the identity

$$
B_{\alpha(u)}\left(z+u, X_{F}\right)=(1-\alpha(u)) \mathbf{1}_{\left\{z+u \geq X_{F}\right\}}-\alpha(u) \mathbf{1}_{\left\{z+u<X_{F}\right\}}
$$

holds $\mathbb{P}$-almost surely for all $u \geq 0$. Thus, $B_{\alpha(u)}\left(z+u, X_{F}\right)+\alpha(u)$ is a Bernoulli random variable with mean $F(z+u)$ that should be greater or equal to $\alpha(u)$ for all $u \geq 0$ provided that $z$ is a good estimate of $\operatorname{LVaR}_{\alpha}(F)$. In particular, we have $F(z+\bar{u})=\alpha(\bar{u})$ for

$$
\bar{u}=\underset{u \geq 0}{\arg \max }\left(\operatorname{VaR}_{\alpha(u)}(F)-u\right) .
$$

Similarly to model selection, we can perform different model validation tests. Using classical methods, we can test whether:

(a) The realizations $B_{\alpha(u)}\left(z+u, x_{1}\right)+\alpha(u), \ldots, B_{\alpha(u)}\left(z+u, x_{n}\right)+\alpha(u)$ are compatible with a Bernoulli distribution with parameter $\alpha(\bar{u})$ if we only test for $u=\bar{u}$.

(b) The realizations $B_{\alpha(u)}\left(z+u, x_{1}\right)+\alpha(u), \ldots, B_{\alpha(u)}\left(z+u, x_{n}\right)+\alpha(u)$ are compatible with a Bernoulli distribution with parameter greater or equal to $\alpha(u)$ if we test for a class of $u \geq 0$.

\section{Applications}

In this section we discuss some applications of LVaR with a special focus on comparing the associated capital requirements with those induced by VaR, ES, and RVaR.

\section{Choosing the benchmark loss distribution}

As highlighted in the introduction, the definition of LVaR allows full flexibility in the choice of the benchmark loss distribution. While the specific choice will clearly depend on the specific context, we describe here two general classes of benchmark loss distributions that are suitable in a variety of financial applications.

A natural choice for the benchmark function $\alpha$ is to consider a step function of the form

$$
\alpha(u)= \begin{cases}\alpha_{1} & \text { if } u_{1} \leq u<u_{2} \\ \alpha_{2} & \text { if } u_{2} \leq u<u_{3} \\ \vdots & \vdots \\ \alpha_{n} & \text { if } u \geq u_{n}\end{cases}
$$


for some critical loss levels $0=u_{1}<\cdots<u_{n}$ and some probability weights $0<\alpha_{1}<\cdots<$ $\alpha_{n} \leq 1$. In this case, we know that

$$
\operatorname{LVaR}_{\alpha}(X)=\max _{k=1, \ldots, n}\left(\operatorname{VaR}_{\alpha_{k}}(X)-u_{k}\right)
$$

This class of benchmark functions have natural applications in the area of capital adequacy and solvency regulation, where $X$ represents the (negative of the) equity value of a certain balance sheet. In this context, one could take $\alpha_{1}$ to be a standard regulatory confidence level, say 0.99 , and add a capital buffer on top of $\mathrm{VaR}_{\alpha_{1}}$ by choosing more conservative confidence levels in correspondence to the critical loss thresholds $u_{2}, \ldots, u_{n}$, which may be determined, for instance, as increasing fractions of some pre-specified target capital level, e.g. the level of available capital. Since $\mathrm{LVaR}_{\alpha}$ produces higher capital requirements than $\mathrm{VaR}_{\alpha_{1}}$, one could in principle accept even a lower benchmark level $\alpha_{1}$, say 0.95 , and incorporate the regulatory standard only beyond a certain critical loss threshold, which should be approved by regulators and could be company-specific.

The above step function is also natural in portfolio management, where $X$ represents the (negative of the) return of a given portfolio. In this case, the critical loss levels $u_{2}, \ldots, u_{n}$ may be determined as increasing fractions of the capital invested at inception and the $\alpha$ levels would reflect the risk appetite of the investor, e.g. as a result of a questionnaire of the form

(Q1) I accept a loss in no more than ... days out of 100.

(Q2) I accept a loss greater than $u_{2}$ in no more than ... days out of 100 .

(Qn) I accept a loss greater than $u_{n}$ in no more than ... days out of 100.

Another natural choice is to specify $\alpha$ as the tail distribution of some benchmark random loss $L$ and set

$$
\alpha(u)=\mathbb{P}(L \leq u), \quad u \geq 0 .
$$

In this case, the acceptability condition imposed by $\mathrm{LVaR}_{\alpha}$ is equivalent to requiring that the loss distribution of $X$ dominates that of $L$ in the first stochastic order and $\operatorname{LVaR}_{\alpha}(X)$ can be conveniently expressed as

$$
\operatorname{LVaR}_{\alpha}(X)=\sup _{p \in[\alpha(0), 1)}\left(\operatorname{VaR}_{p}(X)-\operatorname{VaR}_{p}(L)\right)
$$

Depending on the specific application, one may select a loss profile that is more or less heavytailed. For instance, the tail of the target loss $L$ might display a (generalized) Pareto distribution with possibly infinite mean in the context of large insurance claims or operational risk but rather a Gaussian or Student distribution in the context of market risk. In the latter case, $\alpha$ could coincide with the loss distribution of a given benchmark portfolio. We show the points discussed above in some concrete examples.

\section{Capital adequacy}

Consider a financial institution with available capital $C_{0}>0$. The capital at the end of a prespecified time period is represented by a positive random variable $C_{1} \in \mathcal{X}$. Here, capital refers 
to the book equity value of the company, i.e. the value of assets net of liabilities. In line with our sign convention, it is natural to focus on the position

$$
X=-C_{1} .
$$

We assume that $C_{0}=100$ and consider the piecewise constant benchmark loss distribution

$$
\alpha(u)= \begin{cases}0.995 & \text { if } 0 \leq u<\frac{1}{20} C_{0} \\ 0.999 & \text { if } \frac{1}{20} C_{0} \leq u<\frac{1}{10} C_{0} \\ 0.9995 & \text { if } u \geq \frac{1}{10} C_{0}\end{cases}
$$

The position $X$ is acceptable with respect to $\alpha$ provided that

$$
\mathbb{P}(X \leq 0) \geq 99.5 \%, \quad \mathbb{P}\left(X \leq \frac{1}{20} C_{0}\right) \geq 99.9 \%, \quad \mathbb{P}\left(X \leq \frac{1}{10} C_{0}\right) \geq 99.95 \% .
$$

The first condition tells us that, in line with the Solvency 2 standards, a default is tolerated in no more than $0.5 \%$ of cases. The other conditions prescribe a maximal probability level for defaults of different magnitudes. In particular, regulators are setting an upper bound of $0.1 \%$, respectively $0.05 \%$, for the probability of incurring a default that is higher than $5 \%$, respectively $10 \%$, of initial capital.

In what follows we assume that $p=\mathbb{P}(X>0)>0.5 \%$ so that $X$ is not acceptable according to $\alpha$ and thus $\operatorname{LVaR}_{\alpha}(X)=\operatorname{LVaR}_{\alpha}\left(X^{+}\right)$by surplus invariance subject to positivity; see Proposition 4.3. Our focus is on the following loss distributions: $F_{X^{+}}(0)=1-p$ and

(a) $F_{X^{+}}(u)=1-p e^{-\lambda u}$ for $u>0$

(b) $F_{X^{+}}(u)=1-p\left(1-\Phi\left(\frac{\log (u)-\mu}{\sigma}\right)\right)$ for $u>0$

for $\lambda>0$ and for $\mu \in \mathbb{R}$ and $\sigma>0$, where $\Phi$ is a standard normal distribution. The above specifications yield

(a) $\mathbb{P}(X \leq u \mid X>0)=1-e^{-\lambda u}$ for $u>0$ (in particular $\mathbb{E}[X \mid X>0]=\frac{1}{\lambda}$ ),

(b) $\mathbb{P}(X \leq u \mid X>0)=\Phi\left(\frac{\log (u)-\mu}{\sigma}\right)$ for $u>0$ (in particular $\left.\mathbb{E}[X \mid X>0]=e^{\mu+\sigma^{2} / 2}\right)$.

Now, take $1-p<s<t<1$ and observe that $\operatorname{VaR}_{1-p}(X)>0$ implies $\operatorname{VaR}_{s}(X)=\operatorname{VaR}_{s}\left(X^{+}\right)$ as well as $\operatorname{RVaR}_{s, t}(X)=\operatorname{RVaR}_{s, t}\left(X^{+}\right)$and $\mathrm{ES}_{s}(X)=\mathrm{ES}_{s}\left(X^{+}\right)$. In the exponential case (a), standard computations show that

- $\operatorname{VaR}_{s}(X)=-\frac{1}{\lambda} \log \left(\frac{1-s}{p}\right)$,

- $\operatorname{RVaR}_{s, t}(X)=\frac{1}{\lambda(t-s)}\left((1-t)\left(\log \left(\frac{1-t}{p}\right)-1\right)-(1-s)\left(\log \left(\frac{1-s}{p}\right)-1\right)\right)$,

- $\operatorname{ES}_{s}(X)=-\frac{1}{\lambda}\left(\log \left(\frac{1-s}{p}\right)-1\right)$.

Similarly, in the lognormal case (b) we have

- $\operatorname{VaR}_{s}(X)=e^{\mu+\sigma \Phi^{-1}\left(1-\frac{1-s}{p}\right)}$,

- $\operatorname{RVaR}_{s, t}(X)=\frac{p}{t-s} e^{\mu+\frac{1}{2} \sigma^{2}}\left(f_{\sigma}\left(1-\frac{1-t}{p}\right)-f_{\sigma}\left(1-\frac{1-s}{p}\right)\right)$,

- $\mathrm{ES}_{s}(X)=\frac{p}{1-s} e^{\mu+\frac{1}{2} \sigma^{2}}\left(1-f_{\sigma}\left(1-\frac{1-s}{p}\right)\right)$, 
where $f_{\sigma}(s):=\Phi\left(\Phi^{-1}(s)-\sigma\right)$ for all $s \in(0,1)$.

In Table 1 we display capital requirements based on LVaR as well as on VaR, RVaR, and ES. The default probability $p=\mathbb{P}(X>0)$ is set at $2 \%$. In the exponential case we let the intensity $\lambda$ vary and in the lognormal case we fix $\mu$ and let the log-volatility $\sigma$ vary. First of all, we observe that $\mathrm{LVaR}_{\alpha}$ is always more conservative than $\mathrm{VaR}_{\underline{\alpha}}$. This is in line with Proposition 3.7. In both cases we see that $\operatorname{LVaR}_{\alpha}(X)$ becomes more and more distant from $\operatorname{VaR}_{\underline{\alpha}}(X)$ as the volatility of $X$ increases. It is clear that there is no structural ordering between $\operatorname{LVaR}_{\alpha}$ and $\mathrm{RVaR}_{\underline{\alpha}, \bar{\alpha}}$ as well as between $\mathrm{LVaR}_{\alpha}$ and $\mathrm{ES}_{\underline{\alpha}}$. The comparison with $\mathrm{RVaR}_{\underline{\alpha}, \bar{\alpha}}$ is particularly interesting because both risk metrics are bounded by the same quantile levels. The computations indicate that $\operatorname{LVaR}_{\alpha}(X)$ becomes greater than $\operatorname{RVaR}_{\alpha, \bar{\alpha}}(X)$ once the volatility of $X$ exceeds a certain threshold. In this sense, it seems that $\mathrm{LVaR}_{\alpha}$ reacts to tail risk in a more sensitive way than $\operatorname{RVaR}_{\underline{\alpha}, \bar{\alpha}}$. When comparing $\operatorname{LVaR}_{\alpha}$ with $\operatorname{ES}_{\underline{\alpha}}$ one should bear in mind that $\operatorname{LVaR}_{\alpha}(X)$ discards all the outcomes of $X$ beyond the bound $\operatorname{VaR}_{\bar{\alpha}}(X)$ whereas $\operatorname{ES}_{\underline{\alpha}}(X)$ takes into account the entire $\bar{\alpha}$-tail of $X$. In spite of this, we observe that

$$
\operatorname{LVaR}_{\alpha}(X)>\operatorname{ES}_{\underline{\alpha}}(X)
$$

as soon as the volatility of $X$ exceeds a certain threshold. This is especially interesting because (7) implies that the capital requirement $\operatorname{ES}_{\underline{\alpha}}(X)$ does not suffice to make $X$ acceptable with respect to $\alpha$. This happens, for instance, in the exponential case (a) with $\lambda=0.1$, which corresponds to an expected default $\mathbb{E}[X \mid X>0]$ equal to $10 \%$ of initial capital, or in the lognormal case (b) with $\sigma=2$, which corresponds to an expected default $\mathbb{E}[X \mid X>0]$ equal to $7 \%$ of initial capital.

\begin{tabular}{|c|c|c|c|c|c|c|}
\hline & $X$ & $\operatorname{VaR}_{\underline{\alpha}}(X)$ & $\operatorname{LVaR}_{\alpha}(X)$ & $\operatorname{RVaR}_{\underline{\alpha}, \bar{\alpha}}(X)$ & $\operatorname{ES}_{\underline{\alpha}}(X)$ & $\operatorname{VaR}_{\bar{\alpha}}(X)$ \\
\hline \multirow{4}{*}{ (a) } & $\lambda=1.0$ & $1 . \overline{39}$ & 1.39 & 2.13 & 2.39 & 3.69 \\
\hline & $\lambda=0.5$ & 2.77 & 2.77 & 4.26 & 4.77 & 7.38 \\
\hline & $\lambda=0.2$ & 6.93 & 9.98 & 10.65 & 11.93 & 18.44 \\
\hline & $\lambda=0.1$ & 13.86 & 26.89 & 21.30 & 23.86 & 36.89 \\
\hline \multirow{4}{*}{ (b) } & $\sigma=0.5$ & 1.40 & 1.40 & 1.81 & 1.95 & 2.66 \\
\hline & $\sigma=1.0$ & 1.96 & 1.96 & 3.36 & 4.14 & 7.10 \\
\hline & $\sigma=1.5$ & 2.75 & 8.91 & 6.47 & 9.80 & 18.92 \\
\hline & $\sigma=2.0$ & 3.85 & 40.40 & 12.86 & 26.82 & 50.40 \\
\hline
\end{tabular}

Table 1: Capital requirements for $p=2 \%$ and $\mu=0$.

\section{Portfolio risk management}

Consider an investor who wants to assess the "risk" of some investment, e.g. in a portfolio of financial assets, by looking at the corresponding profit-and-loss $V_{1}-V_{0}$, where $V_{0}>0$ is the wealth invested at inception and $V_{1} \in \mathcal{X}$ is the wealth at the end of a pre-specified time period. In line with our sign convention, it is natural to focus on the position

$$
X=V_{0}-V_{1} \text {. }
$$

The (relative) investment return can be expressed in terms of $X$ as

$$
R=\frac{V_{1}-V_{0}}{V_{0}}=-\frac{X}{V_{0}} .
$$


We assume that $V_{0}=100$ and consider the piecewise constant benchmark loss distribution

$$
\alpha(u)= \begin{cases}0.9 & \text { if } 0 \leq u<\frac{1}{100} V_{0} \\ 0.95 & \text { if } \frac{1}{100} V_{0} \leq u<\frac{1}{20} V_{0} \\ 0.99 & \text { if } \frac{1}{20} V_{0} \leq u<\frac{1}{10} V_{0} \\ 0.999 & \text { if } u \geq \frac{1}{10} V_{0}\end{cases}
$$

The position $X$ is acceptable with respect to $\alpha$ provided that

$$
\mathbb{P}(X \leq 0) \geq 90 \%, \quad \mathbb{P}\left(X \leq \frac{1}{100} V_{0}\right) \geq 95 \%, \quad \mathbb{P}\left(X \leq \frac{1}{20} V_{0}\right) \geq 99 \%, \quad \mathbb{P}\left(X \leq \frac{1}{10} V_{0}\right) \geq 99.9 \% .
$$

The first condition tells us that losses are tolerated in no more than $10 \%$ of cases. The other conditions prescribe a maximal probability level for losses of different magnitudes. In particular, the agent is setting an upper bound of $5 \%$ (respectively $1 \%$ and $0.1 \%$ ), for the probability of losing more than $1 \%$ (respectively $5 \%$ and $10 \%$ ) of initial wealth. The above conditions can be equivalently expressed in terms of returns as

$$
\mathbb{P}(R \geq 0) \geq 90 \%, \quad \mathbb{P}(R \geq-1 \%) \geq 95 \%, \quad \mathbb{P}(R \geq-5 \%) \geq 99 \%, \quad \mathbb{P}(R \geq-10 \%) \geq 99.9 \% .
$$

According to the first condition, a negative return is accepted in no more than $10 \%$ of the cases. The other conditions prescribe a maximal probability level for negative returns of different magnitudes. The benchmark loss distribution $\alpha$ is therefore chosen to reflect the risk attitude of the agent with respect to investment shortfalls.

We aim to compute $\operatorname{LVaR}_{\alpha}(X)$ and compare it with other risk metrics. In the sequel, we focus on two simple stylized cases, namely

(a) $X=\mu+\sigma Z$ with $Z \sim N(0,1)$

(b) $X=\mu+\sigma T$ with $T \sim t(\nu)$

for $\mu \in \mathbb{R}$ and $\sigma>0$, where $N(0,1)$ denotes the class of standard normal distributions and $t(\nu)$ that of Student's t-distributions with $\nu$ degrees of freedom. In both cases, explicit expressions for $\mathrm{VaR}$ and $\mathrm{RVaR}$ are available. Indeed, let $\Phi$, respectively $\Psi_{\nu}$, be the distribution function of a standard normal, respectively t-Student, distribution. Moreover, let $\phi=\Phi^{\prime}$ and $\psi_{\nu}=\Psi_{\nu}^{\prime}$ be the corresponding density functions. Easy calculations show that, for $0<s<t<1$, we have

- $\operatorname{VaR}_{s}(X)=\mu+\sigma \Phi^{-1}(s)$

- $\operatorname{RVaR}_{s, t}(X)=\mu+\sigma \frac{\phi\left(\Phi^{-1}(s)\right)-\phi\left(\Phi^{-1}(t)\right)}{t-s}$

- $\operatorname{ES}_{s}(X)=\mu+\sigma \frac{\phi\left(\Phi^{-1}(s)\right)}{1-s}$

in the normal case (a) and

- $\operatorname{VaR}_{s}(X)=\mu+\sigma \Psi_{\nu}^{-1}(s)$

- $\operatorname{RVaR}_{s, t}(X)=\mu+\frac{\sigma}{\nu-1} \frac{g_{\nu}(s)-g_{\nu}(t)}{t-s}$

- $\mathrm{ES}_{s}(X)=\mu+\frac{\sigma}{\nu-1} \frac{g_{\nu}(s)}{1-s}$ 
in the Student case (b), where $g_{\nu}(s)=\psi_{\nu}\left(\Psi_{\nu}^{-1}(s)\right)\left(\nu+\left(\Psi_{\nu}^{-1}(s)\right)^{2}\right)$ for all $s \in(0,1)$.

In Table 2 we let the volatility level $\sigma$ vary but fix $\mu=-2$, which is equivalent to setting $\mathbb{E}[R]=2 \%$. The computation with respect to different expected return levels is straightforward by virtue of cash-additivity. We observe that, as expected, $\mathrm{LVaR}_{\alpha}$ is always as conservative as $\operatorname{VaR}_{\alpha}$. The two risk measures coincide if the volatility level is low but get more and more far away as the volatility level increases. This phenomenon is more pronounced in case (b) due to the fact that a t-Student distribution has heavier tails than a normal distribution. It is clear from Table 2 that neither $\operatorname{LVaR}_{\alpha}$ nor $\mathrm{RVaR}_{\underline{\alpha}, \bar{\alpha}}$ dominates each other in a systematic way. In this example, $\operatorname{LVaR}_{\alpha}(X)$ is less stringent than $\operatorname{RVaR}_{\underline{\alpha}, \bar{\alpha}}(X)$ when $X$ is mildly risky and becomes more conservative as the volatility level exceeds a certain threshold. Once again, this can be appreciated in a more pronounced form in case (b). As in the previous example on capital adequacy, the above computations would then seem to indicate that $\mathrm{LVaR}_{\alpha}$ reacts to tail risk in a more sensitive way than $\mathrm{RVaR}_{\underline{\alpha}, \bar{\alpha}}$. It is interesting to observe that $\mathrm{LVaR}_{\alpha}$ may be more conservative than $\mathrm{ES}_{\underline{\alpha}}$ as well. In this case, setting a capital reserve equal to $\mathrm{ES}_{\underline{\alpha}}$ does not allow to fulfill the acceptability requirement imposed by $\alpha$ on the loss distribution.

\begin{tabular}{|c|c|c|c|c|c|c|}
\hline$X$ & $\sigma$ & $\operatorname{VaR}_{\underline{\alpha}}(X)$ & $\operatorname{LVaR}_{\alpha}(X)$ & $\mathrm{RVaR}_{\underline{\alpha}, \bar{\alpha}}(X)$ & $\mathrm{ES}_{\underline{\alpha}}(X)$ & $\operatorname{VaR}_{\bar{\alpha}}(X)$ \\
\hline & 1 & -0.72 & -0.72 & -0.26 & -0.25 & 1.09 \\
& 2.5 & 1.20 & 1.20 & 2.35 & 2.39 & 5.73 \\
(a) & 5 & 4.41 & 5.22 & 6.69 & 6.77 & 13.45 \\
& 7.5 & 7.61 & 11.18 & 11.04 & 11.16 & 21.18 \\
& 10 & 10.82 & 18.90 & 15.39 & 15.55 & 28.90 \\
\hline & 1 & -0.52 & -0.52 & 0.25 & 0.30 & 3.89 \\
& 2.5 & 1.69 & 2.73 & 3.62 & 3.76 & 12.73 \\
(b) & 5 & 5.38 & 17.47 & 9.25 & 9.51 & 27.47 \\
& 7.5 & 9.07 & 32.20 & 14.87 & 15.27 & 42.20 \\
& 10 & 12.76 & 46.93 & 20.50 & 21.02 & 56.93 \\
\hline
\end{tabular}

Table 2: Risk measures for $\mu=-2$ and $\nu=5$.

\section{Catastrophic risk}

As illustrated by a number of recent insurance losses caused by natural disasters, catastrophic risk is a major concern for insurance and especially reinsurance companies. Broadly speaking, catastrophic risk refers to low-probability events, such as tsunamis, hurricanes and earthquakes, that may lead to very large insurance claims. In order to fit the corresponding observed losses one typically needs infinite-mean models involving Pareto distributions with tail parameter in the range $[0.5,1]$. The same low-probability but high-severity pattern is encountered in operational risk. In this case, it is standard to employ Pareto distributions with tail parameter in the range [0.5, 5]. For details see McNeil et al. (2015).

With these two applications in mind, we consider a Pareto-tailed benchmark function of the form

$$
\alpha(u)=1-\frac{q}{(1+u)^{\eta}}, \quad u \geq 0,
$$

for $\eta>0$ and $q \in(0,1)$. Here, $\eta$ is the tail parameter of a Pareto distribution and $q=1-\underline{\alpha}$ is interpreted as the maximal tolerated loss probability. Note that a position $X$ is acceptable with respect to $\alpha$ whenever

$$
\mathbb{P}(X \leq u) \geq 1-\frac{q}{(1+u)^{\eta}} \quad \text { for every } u \geq 0 .
$$


In particular, we must have $\mathbb{P}(X \leq 0) \geq 1-q$ and the tail of $X$ must be controlled by a Pareto tail with parameter $\eta$. We focus on random losses $X$ with distribution

$$
F_{X}(u)= \begin{cases}1-\frac{p}{(1+u)^{\theta}} & \text { if } u \geq 0 \\ 0 & \text { otherwise }\end{cases}
$$

where $\theta>0$ is a given tail parameter and $p=\mathbb{P}(X>0)$ is the underlying loss probability. In particular, note that

$$
\mathbb{P}(X \leq u \mid X>0)=1-\frac{1}{(1+u)^{\theta}} \quad \text { for every } u>0,
$$

showing that the conditional distribution of $X$ given that a loss will occur is precisely a Pareto distribution with parameter $\theta$. In view of what we said above, the random variable $X$ may represent a large loss due to catastrophic or operational risk. In what follows we assume that $p>q$, which means that $X$ exhibits a higher loss probability than the acceptable one.

It is easy to determine VaR and ES for the loss $X$. In particular, for any $1-p<s<1$ we have

$$
\operatorname{VaR}_{s}(X)=\left(\frac{1-s}{p}\right)^{-\frac{1}{\theta}}-1 \quad \text { and } \quad \operatorname{ES}_{s}(X)= \begin{cases}\frac{\theta}{\theta-1}\left(\frac{1-s}{p}\right)^{-\frac{1}{\theta}}-1 & \text { if } \theta>1 \\ +\infty & \text { if } \theta \leq 1\end{cases}
$$

According to Proposition 3.7, we can therefore express $\operatorname{LVaR}_{\alpha}(X)$ as

$$
\operatorname{LVaR}_{\alpha}(X)=\sup _{u \geq 0}\left(\operatorname{VaR}_{\alpha(u)}(X)-u\right)=\sup _{u \geq 0}\left(\left(\frac{p}{q}\right)^{\frac{1}{\theta}}(1+u)^{\frac{\eta}{\theta}}-(1+u)\right)
$$

where we used that $\alpha(u) \geq 1-q>1-p$ for all $u \geq 0$. It is easy to verify that the objective function in the above optimization problem is convex and strictly increasing for $\theta \leq \eta$ and strictly concave otherwise. In the latter case, it attains a maximum at

$$
u^{*}=\max \left(0,\left(\frac{\eta}{\theta}\right)^{\frac{\theta}{\theta-\eta}}\left(\frac{p}{q}\right)^{\frac{1}{\theta-\eta}}-1\right) .
$$

Table 3 compares capital requirements based on VaR and ES with those based on LVaR for different choices of the tail parameter of $X$. The loss probability of $X$ is taken to be $1 \%$ whereas the maximal tolerated loss probability is set to $0.1 \%$ in accordance to the operational risk standard of Solvency 2. In line with Proposition 3.7, we see that $\mathrm{LVaR}_{\alpha}$ is always more conservative than $\mathrm{VaR}_{\underline{\alpha}}$ and the gap between $\mathrm{LVaR}_{\alpha}$ and $\mathrm{VaR}_{\underline{\alpha}}$ increases as the tail parameter $\theta$ decreases. In other words, $\operatorname{LVaR}_{\alpha}(X)$ becomes more stringent than $\operatorname{VaR}_{\underline{\alpha}}(X)$ as $X$ becomes more heavy-tailed. This is because $\mathrm{LVaR}_{\alpha}$ depends also on the magnitude of losses, not only on their frequency. The comparison between the outcomes of $\mathrm{LVaR}_{\alpha}$ and those of $\mathrm{ES}_{\underline{\alpha}}$ is particularly interesting since both risk measures take into account the entire tail distribution beyond the $\underline{\alpha-}$ quantile. In general, we see that none of the two risk measures is systematically more conservative than the other. As far as finiteness is concerned, it may happen that both fail to be finite or that only one of them does. In particular, the entries corresponding to $\theta \in\{0.5,0.75,1\}$ and $\eta \in\{0.1,0.25,0.5,0.75\}$ exhibit an example of an infinite-mean distribution to which $\mathrm{LVaR}_{\alpha}$ may associate, contrary to $\mathrm{ES}_{\underline{\alpha}}$, a finite capital requirement. Since we have the liberty to gauge the tail parameter $\eta$ depending on the underlying random loss without changing the "regulatory" confidence level $\underline{\alpha}$, the example shows that we can choose $\eta$ small enough, relative to $\theta$, so as to ensure

$$
\operatorname{VaR}_{\underline{\alpha}}(X)<\operatorname{LVaR}_{\alpha}(X)<\infty=\operatorname{ES}_{\underline{\alpha}}(X)
$$

whenever $X$ has infinite mean. In this case, the risk measure $\operatorname{LVaR}_{\alpha}$ could be used as a feasible alternative to $\mathrm{ES}_{\underline{\alpha}}$. 


\begin{tabular}{|c|c|cccccc|c|}
\hline$\theta$ & \multirow{2}{*}{$\operatorname{VaR}_{\underline{\alpha}}(X)$} & $\eta=0.1$ & $\eta=0.25$ & $\eta=0.5$ & $\eta=0.75$ & $\eta=1$ & $\eta=1.5$ & $\operatorname{ES}_{\underline{\alpha}}(X)$ \\
\hline 0.5 & 99 & 169.18 & 2500 & $+\infty$ & $+\infty$ & $+\infty$ & $+\infty$ & $+\infty$ \\
0.75 & 20.54 & 21.96 & 38.49 & 1481.48 & $+\infty$ & $+\infty$ & $+\infty$ & $+\infty$ \\
1 & 9 & 9 & 10.18 & 25 & 1054.69 & $+\infty$ & $+\infty$ & $+\infty$ \\
1.5 & 3.64 & 3.64 & 3.64 & 3.85 & 5.39 & 14.81 & $+\infty$ & 12.92 \\
2 & 2.16 & 2.16 & 2.16 & 2.16 & 2.19 & 2.50 & 10.55 & 5.32 \\
2.5 & 1.51 & 1.51 & 1.51 & 1.51 & 1.51 & 1.51 & 1.86 & 3.19 \\
3 & 1.15 & 1.15 & 1.15 & 1.15 & 1.15 & 1.15 & 1.16 & 2.23 \\
\hline
\end{tabular}

Table 3: Capital requirements for $p=1 \%$ and $q=1-\underline{\alpha}=0.1 \%$.

\section{Conclusions}

In this paper we introduced a class of quantile-based risk measures, called Loss Value at Risk, that capture tail risk by imposing a bound on the probability of exceeding certain pre-specified loss levels. The bound is mathematically described by a so-called benchmark loss distribution. The underlying guiding principle is that higher losses should be tolerated with lower probability. As discussed in the introduction and illustrated by our results, neither VaR nor, more importantly, ES allow to control tail risk in the way described above. Hence, LVaR can be employed in conjunction with VaR and ES to detect to which extent these two standard risk measures underestimate that aspect of tail risk. At the same time, in view of its interpretation as capital requirement rules, LVaR constitutes a natural alternative to VaR and ES. This is particularly true in the presence of heavy-tailed risks with infinite mean. In this case, VaR will typically underestimate risk while ES, being non finite, simply cannot be used as an operational capital adequacy tool. An additional appealing feature of LVaR is that the benchmark loss distribution can be tailored to the reference loss profile of the underlying risk class.

Many questions are nevertheless still open and deserve further investigation. In what follows we highlight three directions of future research. From a practical point of view, it would be necessary to complement the study conducted in Section 6 with an extensive empirical analysis of risk measures based on benchmark loss distributions. In particular, it would be important to assess how different estimation procedures for $\mathrm{VaR}$ affect the level of capital requirements. From a theoretical perspective, a considerable attention has been devoted in the literature to the problem of optimal risk allocation among a certain number of agents. From a mathematical point of view, this problem can be addressed with the help of infimal convolutions, see for instance Barrieu and El Karoui (2005), Filipović and Svindland (2008), Tsanakas (2009), Embrechts et al. (2018). It would be therefore interesting to study infimal convolutions of risk measures based on benchmark loss distributions. Incidentally, this would also provide a full picture on the aforementioned problem of regulatory arbitrage, see Remark 4.7. Finally, we wish to highlight that benchmark loss distributions could be employed to define other classes of risk measures. One possibility that seems promising is when the acceptability condition imposed by LVaR, namely

$$
\operatorname{VaR}_{\alpha(u)}(X)-u \leq 0 \text { for every } u \geq 0,
$$

is required to be satisfied only on average. This idea, which was suggested to us during the review process, would entail a relaxation of the acceptability condition imposed by LVaR that may be useful in practice because a risk manager might decide to ignore violations occurring on a small interval of losses (note that a violation will always take place on a full interval of losses). In principle, when averaging, one could even assign different weights to different loss levels so 
as to give more importance to losses belonging to a pre-specified range.

\section{References}

Acerbi, C. and Szekely, B. (2017). General properties of backtestable statistics. Preprint, SSRN.2905109.

Acerbi, C. and Tasche, D. (2002). On the coherence of expected shortfall. Journal of Banking 83 Finance, 26(7):1487-1503.

Albanese, C. and Lawi, S. (2004). Spectral risk measures for credit portfolios. Risk Measures for the 21st Century, ed. Giorgio Szegö, pages 209-226.

Artzner, P., Delbaen, F., Eber, J.-M., and Heath, D. (1999). Coherent measures of risk. Mathematical Finance, 9(3):203-228.

Artzner, P., Delbaen, F., and Koch-Medina, P. (2009). Risk measures and efficient use of capital. ASTIN Bulletin, 39(1):101-116.

Barrieu, P. and El Karoui, N. (2005). Inf-convolution of risk measures and optimal risk transfer. Finance and Stochastics, 9(2):269-298.

BCBS (2012). Consultative Document May 2012. Fundamental review of the trading book. Basel Committee on Banking Supervision. Basel: Bank for International Settlements.

BCBS (2016). Standards: Minimum capital requirements for market risk. Basel Committee on Banking Supervision. Basel: Bank for International Settlements.

Bellini, F. and Bignozzi, V. (2015). On elicitable risk measures. Quantitative Finance, 15(5):725-733.

Bellini, F. and Di Bernardino, E. (2017). Risk management with expectiles. The European Journal of Finance, 23(6):487-506.

Bellini, F., Negri, I., and Pyatkova, M. (2018). Backtesting VaR and Expectiles with realized scores. Statistical Methods 85 Applications, to appear.

Burzoni, M., Peri, I., and Ruffo, C. M. (2017). On the properties of the Lambda value at risk: robustness, elicitability and consistency. Quantitative Finance, 17(11):1735-1743.

Cont, R., Deguest, R., and He, X. (2013). Loss-based risk measures. Statistics 8 Risk Modeling with Applications in Finance and Insurance, 30(2):133-167.

Cont, R., Deguest, R., and Scandolo, G. (2010). Robustness and sensitivity analysis of risk measurement procedures. Quantitative Finance, 10(6):593-606.

Daníelsson, J., Embrechts, P., Goodhart, C., Keating, C., Muennich, F., Renault, O., and Shin, H. S. (2001). An academic response to Basel II. Special Paper 130, London School of Economics Financial Markets Group.

Delbaen, F., Bellini, F., Bignozzi, V., and Ziegel, J. F. (2016). Risk measures with the CxLS property. Finance and Stochastics, 20:433-453.

Embrechts, P., Liu, H., and Wang, R. (2018). Quantile-based risk sharing. Operations Research, 66(4):936-949.

Embrechts, P., Puccetti, G., Rüschendorf, L., Wang, R., and Beleraj, A. (2014). An Academic Response to Basel 3.5. Risks, 2:25-48.

Farkas, W., Koch-Medina, P., and Munari, C. (2014a). Beyond cash-additive risk measures: when changing the numéraire fails. Finance and Stochastics, 18(1):145-173. 
Farkas, W., Koch-Medina, P., and Munari, C. (2014b). Capital requirements with defaultable securities. Insurance: Mathematics and Economics, 55:58-67.

Filipović, D. and Svindland, G. (2008). Optimal capital and risk allocations for law-and cash-invariant convex functions. Finance and Stochastics, 12(3):423-439.

Fissler, T., Ziegel, J. F., and Gneiting, T. (2016). Expected Shortfall is jointly elicitable with Value at Risk-Implications for backtesting. Risk, pages 58-61.

Föllmer, H. and Schied, A. (2016). Stochastic Finance: An Introduction in Discrete Time. De Gruyter, Berlin, 4th edition.

Frittelli, M., Maggis, M., and Peri, I. (2014). Risk measures on $\mathcal{P}(\mathbb{R})$ and Value at Risk with probability/loss function. Mathematical Finance, 24(3):442-463.

Galichon, A. (2010). The var at risk. International Journal of Theoretical and Applied Finance, 13(04):503-506.

Gneiting, T. (2011). Making and evaluating point forecasts. Journal of the American Statistical Association, 106(494):746-762.

Guldimann, T. (2000). The story of risk metrics. Risk, 13(1):56-58.

Huber, P. (1981). Robust Statistics. Wiley, New York.

IAIS (2014). Consultation Document December 2014. Risk-based global insurance capital standard. International Association of Insurance Supervisors.

Jarrow, R. (2013). Capital adequacy rules, catastrophic firm failure, and systemic risk. Review of Derivatives Research, 16(3):219-231.

Koch-Medina, P., Moreno-Bromberg, S., and Munari, C. (2015). Capital adequacy tests and limited liability of financial institutions. Journal of Banking \& Finance, 51:93-102.

Koch-Medina, P., Munari, C., and Šikić, M. (2017). Diversification, protection of liability holders and regulatory arbitrage. Mathematics and Financial Economics, 11(1):63-83.

Krätschmer, V., Schied, A., and Zähle, H. (2014). Comparative and qualitative robustness for lawinvariant risk measures. Finance and Stochastics, 18(2):271-295.

Liu, F. and Wang, R. (2016). A theory for measures of tail risk. Preprint, SSRN.2841909.

Mao, T. and Wang, R. (2016). Risk aversion in regulatory capital calculation. Preprint, SSRN.2658669.

McNeil, A. J., Frey, R., and Embrechts, P. (2015). Quantitative Risk Management: Concepts, Techniques, Tools. Princeton University Press, Princeton, NJ, Revised edition.

Nolde, N. and Ziegel, J. F. (2017). Elicitability and backtesting: Perspectives for banking regulation. Annals of Applied Statistics, 11(4),1833-1874.

Song, Y. and Yan, J.-A. (2009). Risk measures with comonotonic subadditivity or convexity and respecting stochastic orders. Insurance: Mathematics and Economics, 45(3):459-465.

Staum, J. (2013). Excess invariance and shortfall risk measures. Operations Research Letters, 41(1):47-53.

Tsanakas, A. (2009). To split or not to split: Capital allocation with convex risk measures. Insurance: Mathematics and Economics, 44(2):268-277.

Wang, R. (2016). Regulatory arbitrage of risk measures. Quantitative Finance, 16(3):337-347.

Weber, S. (2018). Solvency II, or how to swipe the downside risk under the carpet. Insurance: Mathematics and economics, 82:191-200. 
Yamai, Y. and Yoshiba, T. (2005). Value-at-risk versus expected shortfall: A practical perspective. Journal of Banking \&3 Finance, 29(4):997-1015.

Ziegel, J. F. (2016). Coherence and elicitability. Mathematical Finance, 26(4):901-918. 